\title{
MODELING SHALLOW OVER-SATURATED MIXTURES ON ARBITRARY RIGID TOPOGRAPHY
}

\author{
I. Luca ${ }^{*}$ \\ C. Y. Kuo \\ Department of Mathematics II \\ University Politehnica of Bucharest \\ Splaiul Independentei 313, 060042 Bucharest, Romania \\ Division of Mechanics, Research Center for Applied Sciences \\ Academia Sinica \\ Taipei, Taiwan 11529, R.O.C.
}

\author{
K. Hutter \\ Bergstrasse 5, 8044 Zürich \\ Switzerland
}

\author{
Y. C. Tai \\ Department of Hydraulic and Ocean Engineering \\ National Cheng Kung University \\ Tainan, Taiwan 70101, R.O.C.
}

\begin{abstract}
In this paper a system of depth-integrated equations for over-saturated debris flows on threedimensional topography is derived. The lower layer is a saturated mixture of density preserving solid and fluid constituents, where the pore fluid is in excess, so that an upper fluid layer develops above the mixture layer. At the layer interface fluid mass exchange may exist and for this a parameterization is needed. The emphasis is on the description of the influence on the flow by the curvature of the basal surface, and not on proposing rheological models of the avalanching mass. To this end, a coordinate system fitted to the topography has been used to properly account for the geometry of the basal surface. Thus, the modeling equations have been written in terms of these coordinates, and then simplified by using (1) the depth-averaging technique and (2) ordering approximations in terms of an aspect ratio $\epsilon$ which accounts for the scale of the flowing mass. The ensuing equations have been complemented by closure relations, but any other such relations can be postulated. For a shallow two-layer debris with clean water in the upper layer, flowing on a slightly curved surface, the equilibrium free surface is shown to be horizontal.
\end{abstract}

Keywords: Two-layer debris flow, Over-saturated mixture, Shallow flow, Fluid mass exchange, Arbitrary topography.

\section{INTRODUCTION}

There are many geophysical flows, such as earthquake, typhoon induced landslides, turbidity currents, which develop into a shallow layer of solid-fluid (e.g. sediment-water) mixture beneath a lighter fluid layer (e.g. clean water). Larcan et al. [1] have evidenced such a stratification in a dam-break experiment of hyperconcentrated mixture of water and granular material, depending on the bed slope and the solid volume fraction. The need to have models for a two-layer debris flow (called immature debris flow by Takahashi [2]), is therefore clear.

There are models in which the lower sediment-water layer is treated as a one-constituent body with its own rheology, Fraccarollo and Capart [3], Morales [4], Luca et al. [5]. Slightly more general, Fernández-Nieto et al. [6] treat the lower layer as a mixture of two con- stituents moving with the same velocity and according to the momentum balance equation of the mixture as a whole; doing so, the stresses of each mixture constituent could be accounted for in this momentum balance equation. All the cited papers consider the two layers as being immiscible. The most comprehensive treatment of an immature debris flow is likely due to Takahama et al. [7], which includes the fluid mass exchange at the layer interface, but still dealing with mixture constituents moving with the same velocity, which excludes the influence of the interaction force between the mixture constituents.

However, equality of the granular and pore-fluid velocities in a sediment-water mixture is a strong assumption. With it a debris can not be initiated by motion of water on a stable debris deposition (such as a dam or soil slope). Moreover, a consolidation process in the debris deposit of a water soaked landslide is equally

\footnotetext{
* Corresponding author (iolucaro@yahoo.com)
} 
impossible to be described. Larcan et al. [1] emphasized the need to have a model for a two-layer debris flow in which each mixture constituent is considered with its own velocity. To our knowledge, except the papers by Berzi et al. [8-10], in which a simple model is proposed for such a flow in a laboratory chute, a general model taking into account the temporal effects has not yet been derived. However, theoretical descriptions of the motion of an ice layer above an ice-sediment layer by Hutter et al. [11], Svendsen et al. [12] and $\mathrm{Wu}$ et al. [13] are quite general: different velocities of ice and sediment in the lower layer, and mass entrainment at the layer interface.

Here we treat the debris as developed into a lower solid-fluid mixture layer, in which each constituent moves according to its own mass and momentum balance equations, and an upper fluid layer, with or without fluid mass transfer at the layer interface. Moreover, noting that the trend in avalanche modeling is to account for curvature effects (see, e.g., Sivakumaran et al. [14], Dewals et al. [15], Iverson [16], Pudasaini et al. $[17,18]$, Bouchut and Westdickenberg [19], De Toni and Scotton [20], Bouchut et al. [21], Tai and Kuo [22], Tai and Lin [23], Pelanti et al. [24], Luca et al. [25-27,5]), emphasis is on the description of the flow on arbitrary terrain, using the approach initiated by Bouchut and Westickenberg [19], and not on the formulation of constitutive laws, even if we indicate such laws as possible choices. In particular, the shallow saturated mixture in the lower layer is treated in Truesdell's sense, in the same manner as it has been done by Luca et al. [27].

In Sect. 2 we recall the change of coordinates as used by Bouchut and Westdickenberg [19] in the neighborhood of the basal topography, and list the properties of this change of coordinates. In Sect. 3 we present the intrinsic modeling equations describing the two-layer flow of debris with fluid mass exchange at the layer interface (miscible layers) and without such a mass entrainment (immiscible layers). Balance statements of mass and momentum are presented in absolute and topography-fitted curvilinear coordinates as are boundary and transition conditions. The equations are made dimensionless, then depth-integrated over the two layers, and subsequently, via a scaling analysis, reduced to shallow flow equations. Closure relations are presented in Sect. 4, and in Sect. 5 a set of model equations in a single spatial dimension are presented. In Sect. 6 we show that, when a shallow over-saturated mixture, with water as the fluid constituent, is at rest on a slightly curved basal topography, the modeling equations predict a horizontal free surface. We end with conclusions in Sect. 7.

Let us introduce notations, which we use throughout the paper. The 2-column matrices are denoted by small upright boldface letters, e.g., a, and the $2 \times 2$ matrices are denoted by capital upright boldface letters, e.g. A. A similar notation, but with slanted letters, e.g., a, $\boldsymbol{A}$, is used for vectors and tensors in the translation vector space $\mathcal{V}$ of the 3D Euclidean space $\mathcal{E}$. The dyadic product of two column matrices $\mathbf{a}$ and $\mathbf{b}$ is $\mathbf{a} \otimes \mathbf{b} \equiv$ $\mathbf{a b}^{T}$ where $T$ stands for the transpose of a matrix; the symbol $\otimes$ also denotes the tensor product of two vec- tors in $\mathcal{V}$. For a square matrix $\mathbf{A}, \operatorname{tr} \mathbf{A}$ is the trace and $\operatorname{det} \mathbf{A}$ is the determinant of $\mathbf{A}$. The inner product of the 2-column matrices $\mathbf{a}$ and $\mathbf{b}$ is $\mathbf{a} \cdot \mathbf{b} \equiv \operatorname{tr}\left(\mathbf{a b}^{T}\right)$, and if $\mathbf{A}, \mathbf{B}$ are square matrices, $\mathbf{A} \cdot \mathbf{B} \equiv \operatorname{tr}\left(\mathbf{A B}^{T}\right)$. Greek indices have the values 1,2 , Latin indices range from 1 to 3 , and summation over repeated indices is understood. The matrix I denotes the $2 \times 2$ unit matrix, $\delta_{\beta}^{\alpha}$ is the Kronecker symbol, and $\mathbf{1}$ is the unit second order tensor on $\mathcal{V}$. For a scalar function $f$ and a 2-column matrix function $\mathbf{v}=\left(v^{1}, v^{2}\right)^{T}$ depending on the Cartesian coordinates $x_{1}, x_{2}$ (Fig. 1), we let grad denote the gradient operator, i.e.,

$$
\operatorname{grad} f \equiv\left(\frac{\partial f}{\partial x_{1}}, \frac{\partial f}{\partial x_{2}}\right)^{T}, \quad \operatorname{grad} \mathbf{v} \equiv\left(\frac{\partial v^{\alpha}}{\partial x_{\beta}}\right),
$$

and if $f, \mathbf{v}$ depend on the curvilinear coordinates $\xi^{1}, \xi^{2}$, we use Grad, i.e.,

$$
\operatorname{Grad} f \equiv\left(\frac{\partial f}{\partial \xi^{1}}, \frac{\partial f}{\partial \xi^{2}}\right)^{T}, \quad \operatorname{Grad} \mathbf{v} \equiv\left(\frac{\partial v^{\alpha}}{\partial \xi^{\beta}}\right) .
$$

Notation Div stands for the divergence operator referring to $\xi^{1}, \xi^{2}$,

$$
\operatorname{Div} \mathbf{v} \equiv \frac{\partial v^{\alpha}}{\partial \xi^{\alpha}}, \quad \operatorname{Div} \mathbf{T} \equiv \frac{\partial T^{\alpha \beta}}{\partial \xi^{\beta}} \mathbf{e}_{\alpha}
$$

$\mathbf{T}$ is a $2 \times 2$-matrix function with $T^{\alpha \beta}$ as entries, and

$$
\mathbf{e}_{1} \equiv(1,0)^{T}, \quad \mathbf{e}_{2} \equiv(0,1)^{T} .
$$

\section{CHANGE OF COORDINATES}

In modeling surface flows on general topography, Bouchut and Westdickenberg [19] and, independently, De Toni and Scotton [20] have used curvilinear coordinates fitted to the topography. For the flow over a spillway this type of coordinates has been also used by Dressler [28], Sivakumaran et al. [14,29] and Dewals et al. [15]. In this paper we use the approach initiated in [19].

We briefly describe the topographic surface. Letting $\mathrm{Ox}_{1} x_{2} x_{3}$ be a Cartesian coordinate system in the 3D Euclidean point space $\mathcal{E}$, such that $O x_{3}$ is (physically) the vertical direction, see Fig. 1, the mathematical model of the land area is a surface $\mathcal{S}$ given parametrically by

$$
\begin{aligned}
& x_{1}=x_{1}\left(\xi^{1}, \xi^{2}\right), x_{2}=x_{2}\left(\xi^{1}, \xi^{2}\right), \\
& x_{3}=b\left(x_{1}\left(\xi^{1}, \xi^{2}\right), x_{2}\left(\xi^{1}, \xi^{2}\right)\right) ;
\end{aligned}
$$

$x_{1}, x_{2}$, and $b$ are functions of class $C^{2}$ on some open subset $\Delta_{0}$ of $\mathbb{R}^{2}$, and

$$
\operatorname{det} \mathbf{F}>0, \quad \mathbf{F} \equiv\left(\frac{\partial x_{i}}{\partial \xi^{\alpha}}\right)_{i, \alpha \in\{1,2\}} .
$$

If $\rho$ is the position vector (in the Cartesian coordinate system) of a point on $\mathcal{S}$, then

$$
\tau_{\alpha} \equiv \frac{\partial \rho}{\partial \xi^{\alpha}}, \quad \alpha \in\{1,2\}
$$




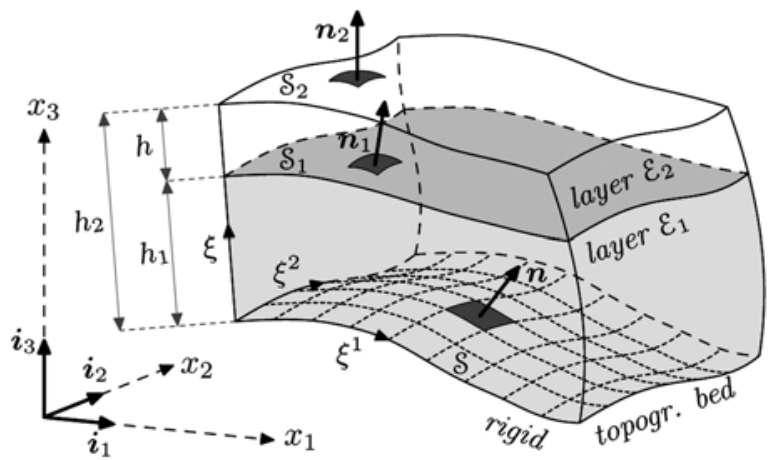

Fig. 1 Geometry of the two-layer flow with layers $\mathcal{E}_{1}$, $\mathcal{E}_{2}$, basal surface $\mathcal{S}$, interface $\mathcal{S}_{1}$ and top surface $\mathcal{S}_{2}$. Layer $\mathcal{E}_{1}$ is filled with a solid-fluid mixture, layer $\mathcal{E}_{2}$ with the same fluid. The bed is treated as rigid

define the natural basis of the tangent space to $\mathcal{S}$ at that point, and a unit normal vector field to $\mathcal{S}$ is

$$
\boldsymbol{n} \equiv \frac{\tau_{1} \times \tau_{2}}{\left\|\tau_{1} \times \tau_{2}\right\|}
$$

here $\times$ stands for the cross product of two vectors, and || || represents the Euclidean norm. Condition (2) implies that $\boldsymbol{n}$ so defined points upwards. We denote the components of $\boldsymbol{n}$ with respect to the Cartesian basis $\left\{\boldsymbol{i}_{1}\right.$, $\left.\boldsymbol{i}_{2}, \boldsymbol{i}_{3}\right\}$ by $\left(-s_{1},-s_{2}, c\right)$, and collect $s_{1}, s_{2}$ in the 2-column matrix $\mathbf{s} \equiv\left(s_{1}, s_{2}\right)^{T}$. We have

$$
c=(1+\operatorname{grad} b \cdot \operatorname{grad} b)^{-1 / 2}, \mathbf{s}=c \operatorname{grad} b .
$$

The coefficients of the first fundamental form of $\mathcal{S}$ are $\phi_{\alpha \beta} \equiv \tau_{\alpha} \cdot \tau_{\beta}$, see (3), and

$$
\left(\phi_{\alpha \beta}\right)=\mathbf{M}_{0}^{-1}, \mathbf{M}_{0} \equiv \mathbf{F}^{-1}(\mathbf{I}-\mathbf{s} \otimes \mathbf{s}) \mathbf{F}^{-T},
$$

holds. By the representation

$$
\frac{\partial \boldsymbol{n}}{\partial \xi^{\beta}}=-b_{\alpha \beta} \tau^{\alpha}=-W_{\beta}^{\alpha} \tau_{\alpha}, \beta \in\{1,2\},
$$

with $\left\{\tau^{1}, \tau^{2}\right\}$ the reciprocal basis of $\left\{\tau_{1}, \tau_{2}\right\}$, one defines

$$
\mathbf{H} \equiv\left(b_{\alpha \beta}\right), \mathbf{W} \equiv\left(W_{\beta}^{\alpha}\right)
$$

Notice the matrix relations

$$
\mathbf{W}=\mathbf{M}_{0} \mathbf{H}, \quad \mathbf{H}=c \mathbf{F}^{T} \operatorname{grad}(\operatorname{grad} b) \mathbf{F} .
$$

The curvature tensor $\mathcal{H}$ and the mean curvature $\Omega$ at a given point on $\mathcal{S}$ are defined by

$$
\mathcal{H} \equiv b_{\alpha \beta} \tau^{\alpha} \otimes \tau^{\beta}, \Omega \equiv \frac{1}{2} \operatorname{tr} \mathcal{H}=\frac{1}{2} \operatorname{tr} \mathbf{W}
$$

If $\boldsymbol{r}$ is the position vector of a point $P$ lying near the basal surface, and $\rho\left(\xi^{1}, \xi^{2}\right)$ is the position vector of the perpendicular projection onto $\mathcal{S}$ of $P$, the relation $\boldsymbol{r}\left(x_{1}, x_{2}, x_{3}\right)=\boldsymbol{\rho}\left(\xi^{1}, \xi^{2}\right)+\xi \boldsymbol{n}\left(\xi^{1}, \xi^{2}\right) \equiv \boldsymbol{r}\left(\xi^{1}, \xi^{2}, \xi\right)$

defines a change of coordinates in a neighborhood of $\mathcal{S}$, provided the Jacobian $J$ of this transformation is nonzero. Since $J$ is given by

$$
J=\frac{1}{c} \operatorname{det} \mathbf{B} \quad \text { with } \mathbf{B} \equiv \mathbf{F}(\mathbf{I}-\xi \mathbf{W}),
$$

condition $J \neq 0$ is equivalent to

$$
\operatorname{det}(\mathbf{I}-\xi \mathbf{W}) \neq 0
$$

This gives the constraint on the "arbitrariness" of the topography. The vectors

$$
\begin{aligned}
& \boldsymbol{g}_{\beta} \equiv \frac{\partial \boldsymbol{r}}{\partial \xi^{\beta}}=\left(\delta_{\beta}^{\alpha}-\xi W_{\beta}^{\alpha}\right) \tau_{\alpha}, \beta \in\{1,2\} \\
& \boldsymbol{g}_{3} \equiv \frac{\partial \boldsymbol{r}}{\partial \xi}=\boldsymbol{n}
\end{aligned}
$$

define the natural basis of $\mathcal{V}$ at $P$. Remarks:

1. To work with the components of vector and tensor fields in block matrix form, as initiated in [19], corresponding to a vector $\boldsymbol{v}=v^{i} \boldsymbol{g}_{i}$ and a symmetric tensor $\boldsymbol{\sigma}=T^{i j} \boldsymbol{g}_{i} \otimes \boldsymbol{g}_{j}$, the following quantities are introduced:

$$
\begin{aligned}
& \mathbf{v} \equiv\left(v^{1}, v^{2}\right)^{T}, \quad \mathrm{v} \equiv v^{3}, \\
& \mathbf{T} \equiv\left(\begin{array}{ll}
T^{11} & T^{12} \\
T^{12} & T^{22}
\end{array}\right), \quad \mathbf{t} \equiv\left(\begin{array}{l}
T^{13} \\
T^{23}
\end{array}\right) .
\end{aligned}
$$

2. Equation (12) shows that $\boldsymbol{g}_{1}, \boldsymbol{g}_{2}$ are parallel to $\mathcal{S}$, and $\boldsymbol{g}_{3}$ is normal to $\mathcal{S}$. Thus, see (13), we speak of the tangential components $\mathbf{v}$ and the normal component $\mathrm{v}$ of $v$; similarly, if $\sigma$ is the stress tensor, $T^{11}, T^{22}$ are the normal stresses parallel to the base, $T^{33}$ is the normal stress, $T^{12}$ is the shear stress in the tangential direction, and $\mathbf{t}$ gives the shear stresses in the normal direction.

3. If the stress tensor $\sigma$ is written as

$$
\boldsymbol{\sigma}=-p 1+\boldsymbol{\sigma}_{E}
$$

with $p$ the pressure and $\sigma_{E}$ the extra-stress tensor, we have

$$
\mathbf{T}=-p \mathbf{M}+\mathbf{P}, \mathbf{t}=\mathbf{p}, T^{33}=-p+P^{33},
$$

where $\mathbf{T}, \mathbf{t}, T^{33}$ refer to $\sigma$, and $\mathbf{P}, \mathbf{p}, P^{33}$ refer to $\boldsymbol{\sigma}_{E}$, according to (13); $\mathbf{M}$ is

$$
\mathbf{M} \equiv \mathbf{B}^{-1}(\mathbf{I}-\mathbf{s} \otimes \mathbf{s}) \mathbf{B}^{-T}
$$

and note $\mathbf{M}_{0}=\mathbf{M} \mid \xi=0$. 


\section{SHALLOW FLOW OF AN OVER-SATURATED MIXTURE}

The flowing body is considered as consisting of a granular material and a lighter fluid, say water ${ }^{1}$. The fluid is assumed to be in excess, so that the avalanching mass develops into two layers - the lower layer $\mathcal{E}_{1}$, in which granular solid and fluid coexist, and the upper layer $\mathcal{E}_{2}$, in which solely the fluid is present, see Fig. 1 . Fluid mass may be exchanged between the layers.

\subsection{Intrinsic Modeling Equations}

The basic equations at hand are as follows.

(1) In the lower layer the solid-fluid mixture consists of granular and fluid material treated as continuous bodies, moving according to the mass and momentum balances, in which the dynamic interaction is accounted for by means of the so-called interaction force, denoted here by $\stackrel{+}{\boldsymbol{m}}$. Thus, for the solid constituent, characterized by $\rho_{s}$ (mass of the solid constituent per mixture volume), the velocity $\boldsymbol{v}$ and the stress tensor $\sigma_{s}$ (see Table 1 for notations), mass and momentum balance equations are

$$
\begin{aligned}
& \frac{\partial \rho_{s}}{\partial t}+\operatorname{div}\left(\rho_{s} \boldsymbol{v}\right)=0, \\
& \frac{\partial \rho_{s} \boldsymbol{v}}{\partial t}+\operatorname{div}\left(\rho_{s} \boldsymbol{v} \otimes \boldsymbol{v}-\boldsymbol{\sigma}_{s}\right)=\rho_{s} \boldsymbol{b}+\stackrel{+}{\boldsymbol{m}},
\end{aligned}
$$

where $\boldsymbol{b}$ is the specific body force (here gravity acceleration) and div is the spatial divergence operator. Similarly, for the fluid constituent, characterized by $\rho_{f}$ (mass of the fluid constituent per mixture volume), the velocity $\boldsymbol{u}$ and the stress tensor $\sigma_{f}$ obey the mass and momentum balances

$$
\begin{aligned}
& \frac{\partial \rho_{f}}{\partial t}+\operatorname{div}\left(\rho_{f} \boldsymbol{u}\right)=0, \\
& \frac{\partial \rho_{f} \boldsymbol{u}}{\partial t}+\operatorname{div}\left(\rho_{f} \boldsymbol{u} \otimes \boldsymbol{u}-\sigma_{f}\right)=\rho_{f} \boldsymbol{b}-\stackrel{+}{\boldsymbol{m}},
\end{aligned}
$$

where the opposite of $\stackrel{+}{\boldsymbol{m}}$ has been taken in (17). Moreover, we assume that (i) the constituents are density preserving, that is, the true densities $\tilde{\rho}_{s}$, $\tilde{\rho}_{f}$ (mass of a constituent per volume of that constituent) are constant in time and uniform in space, and that (ii) the mixture is saturated, that is, there is no void space within the mixture. If $v$ is the solid volume fraction (volume of the solid constituent per volume of the mixture), we therefore have

$$
\rho_{s}=v \tilde{\rho}_{s}, \quad \rho_{f}=(1-v) \tilde{\rho}_{f}, v \in(0,1),
$$

which transforms Eqs. (16), (17) into

\begin{tabular}{|c|c|}
\hline \multicolumn{2}{|c|}{ layer $\mathcal{E}_{1}$ (lower layer) } \\
\hline $\begin{array}{c}\text { solid constituent } \\
\rho_{s}=v \tilde{\rho}_{s}, v, \sigma_{s} \\
\tilde{\rho}_{s}=\text { constant }\end{array}$ & $\begin{array}{c}\text { fluid constituent } \\
\rho_{f}=(1-v) \tilde{\rho}_{f}, \boldsymbol{u}, \sigma_{f} \\
\tilde{\rho}_{f}=\text { constant }\end{array}$ \\
\hline \multicolumn{2}{|c|}{$\begin{array}{c}\rho_{1} \equiv \rho_{s}+\rho_{f} \\
\boldsymbol{v}_{1} \equiv c_{s} \boldsymbol{v}+\left(1-c_{s}\right) \boldsymbol{u}, \quad c_{s} \equiv \rho_{s} / \rho_{1} \\
\sigma_{1} \equiv \sigma_{s}+\sigma_{f}+\rho_{1} \boldsymbol{v}_{1 \otimes} \boldsymbol{v}_{1}-\rho_{s} \boldsymbol{v} \otimes \boldsymbol{v}-\rho_{f} \boldsymbol{u} \otimes \boldsymbol{u}\end{array}$} \\
\hline \multicolumn{2}{|c|}{ layer $\mathcal{E}_{2}$ (upper layer) } \\
\hline
\end{tabular}

\footnotetext{
1 The pore fluid here may also be considered to be a slurry, i.e., a fluid with suspended clay particles.
}

Table 1 Notations for densities, velocity vectors and stress tensors in the two-layer system

$$
\begin{aligned}
& \frac{\partial v}{\partial t}+\operatorname{div}(u v)=0, \\
& \frac{\partial v \boldsymbol{v}}{\partial t}+\operatorname{div}\left\{v \boldsymbol{v} \otimes \boldsymbol{v}-\frac{1}{\tilde{\rho}_{s}} \boldsymbol{\sigma}_{s}\right\}=v \boldsymbol{b}+\frac{1}{\tilde{\rho}_{s}} \stackrel{\boldsymbol{m}}{ },
\end{aligned}
$$

and, respectively,

$$
\begin{aligned}
& \frac{\partial(1-v)}{\partial t}+\operatorname{div}\{(1-v) \boldsymbol{u}\}=0, \\
& \frac{\partial(1-v) \boldsymbol{u}}{\partial t}+\operatorname{div}\left\{(1-v) \boldsymbol{u} \otimes \boldsymbol{u}-\frac{1}{\tilde{\rho}_{f}} \boldsymbol{\sigma}_{f}\right\}=(1-v) \boldsymbol{b}-\frac{1}{\tilde{\rho}_{f}} \stackrel{+}{ },
\end{aligned}
$$

(2) In the upper layer the fluid is characterized by the density $\tilde{\rho}_{f}$ (we assumed the same fluid in both layers), the velocity $\boldsymbol{v}_{2}$ and the stress tensor $\sigma_{2}$, and moves according to mass and momentum balances

$$
\begin{aligned}
& \operatorname{div} \boldsymbol{v}_{2}=0, \\
& \frac{\partial \boldsymbol{v}_{2}}{\partial t}+\operatorname{div}\left\{\boldsymbol{v}_{2} \otimes \boldsymbol{v}_{2}-\frac{1}{\tilde{\rho}_{f}} \boldsymbol{\sigma}_{2}\right\}=\boldsymbol{b} .
\end{aligned}
$$

(3) The basal surface $\mathcal{S}, x_{3}-b\left(x_{1}, x_{2}\right)=0$, is a stagnant material surface (we neglect erosion/deposition), so that the kinematic boundary conditions read

$$
\boldsymbol{v} \cdot \boldsymbol{n}=0, \boldsymbol{u} \cdot \boldsymbol{n}=0 .
$$

Because of computational intricacies the stresses at the base will be postulated in Sect. 4.

(4) The layer interface $\mathcal{S}_{1}, F_{1}\left(x_{1}, x_{2}, x_{3}, t\right)=0$, is the surface separating the lower layer from the upper layer (we choose $F_{1}$ such that $n_{1}=$ $\nabla F_{1} /\left\|\nabla F_{1}\right\|$ points into the upper layer). More exactly, $\mathcal{S}_{1}$ is the upper boundary of the granular material $\left(\mathcal{S}_{1}\right.$ is material with respect to the solid constituent), which implies

$$
\frac{\partial F_{1}}{\partial t}+\nabla F_{1} \cdot \boldsymbol{v}=0
$$

at $F_{1}=0 . \quad$ In particular, (18) shows that the speed $\mathcal{U}_{1}$ of displacement of the interface, defined as

Journal of Mechanics, Vol. 28, No. 3, September 2012 


$$
\mathcal{U}_{1} \equiv-\frac{\partial F_{1}}{\partial t} /\left\|\nabla F_{1}\right\| \quad \text { at } F_{1}=0
$$

is equal to the normal component of the velocity of the solid constituent, i.e., $\mathcal{U}_{1}=\left.\boldsymbol{v}\right|_{F_{1}=0} \cdot \boldsymbol{n}_{1}$.

If $\mathcal{E}_{1}, \mathcal{E}_{2}$ are immiscible layers (say, case (I)), $\mathcal{S}_{1}$ also coincides with the upper boundary of the fluid constituent in $\mathcal{E}_{1}$ and the lower boundary of the fluid in $\mathcal{E}_{2}$. Thus,

$$
\frac{\partial F_{1}}{\partial t}+\nabla F_{1} \cdot \boldsymbol{u}=0, \frac{\partial F_{1}}{\partial t}+\nabla F_{1} \cdot \boldsymbol{v}_{2}=0
$$

hold at $F_{1}=0$ if there is no fluid mass flux across $\mathcal{S}_{1}$. In particular we have $\mathcal{U}_{1}=\left.\boldsymbol{u}\right|_{F_{1}=0} \cdot \boldsymbol{n}_{1}=\left.\boldsymbol{v}_{2}\right|_{F_{1}=0} \cdot \boldsymbol{n}_{1}$. However, if fluid mass transfer into/from the mixture occurs (say, case (II)), the mass balance across $\mathcal{S}_{1}$ for the fluid in both layers must be accounted for:

$$
\begin{aligned}
\llbracket \rho\left(\boldsymbol{v}-\mathcal{U}_{1} \boldsymbol{n}_{1}\right) \rrbracket \cdot \boldsymbol{n}_{1}=0 \Leftrightarrow \\
\tilde{\rho}_{f}\left(\boldsymbol{v}_{2} \cdot \boldsymbol{n}_{1}-\mathcal{U}_{1}\right)=\mathcal{M}_{\text {int }},
\end{aligned}
$$

where

$$
\left.\mathcal{M}_{\text {int }} \equiv \rho_{f}\left(\boldsymbol{u} \cdot \boldsymbol{n}_{1}-\mathcal{U}_{1}\right)\right|_{F_{1}=0}=\left.\rho_{f}(\boldsymbol{u}-\boldsymbol{v}) \cdot \boldsymbol{n}_{1}\right|_{F_{1}=0}
$$

represents the fluid mass flux from the sediment-water mixture layer into the water layer. In $(20)_{1}$, as well as in the forthcoming (22), $\rho, v$ are generic notations for the density and velocity fields.

Conditions (19) are equivalent to (20) and $\mathcal{M}_{\text {int }}=0$. Thus, the forthcoming analysis refers to case (II): The modeling equations for case (I) will consist of the modeling equations for case (II), together with condition $\mathcal{M}_{\text {int }}=0$.

Apart from the jump of the mass across $\mathcal{S}_{1},(20)$, we account for the jump of momentum across $\mathcal{S}_{1}$. We consider the momentum interaction (i) between the pore fluid in $\mathcal{E}_{1}$ and the fluid in $\mathcal{E}_{2}$, and (ii) between the mixture as a whole in $\mathcal{E}_{1}$ and the fluid in $\mathcal{E}_{2}$. For each case the momentum jump across $\mathcal{S}_{1}$ takes the form

$$
\llbracket \sigma-\rho\left(v-\mathcal{U}_{1} \boldsymbol{n}_{1}\right) \otimes\left(v-\mathcal{U}_{1} \boldsymbol{n}_{1}\right) \rrbracket \cdot \boldsymbol{n}_{1}=\mathbf{0} .
$$

This entails a postulate about which part of the traction $\boldsymbol{\sigma}_{2} \boldsymbol{n}_{1}$ in $\mathcal{E}_{2}$ must be accounted for when (22) is used for the constituent fluid alone. A sensible choice is to postulate

$$
\lambda \boldsymbol{\sigma}_{2} \boldsymbol{n}_{1}, \text { with } \lambda=1-v,
$$

which is a constitutive assumption. Our choice for $\lambda$ is a first, obvious suggestion. Thus, by using (23), exploitation of the jump condition (22) for the fluid gives

$$
\sigma_{f} \boldsymbol{n}_{1}=(1-v) \sigma_{2} \boldsymbol{n}_{1}+\mathcal{M}_{i n t}\left(\boldsymbol{u}-\boldsymbol{v}_{2}\right)
$$

at $F_{1}=0$. Then, using (23), (22) for the mixture (see Table 1 for mixture quantities) reduces to the appealing statement

$$
\boldsymbol{\sigma}_{s} \boldsymbol{n}_{1}=\cup \boldsymbol{\sigma}_{2} \boldsymbol{n}_{1}\left(=(1-\lambda) \boldsymbol{\sigma}_{2} \boldsymbol{n}_{1}\right) .
$$

Let us comment on result (24): (24) has also been used by $\mathrm{Wu}$ et al. [13] for the flow of a glacier, however in a special form and as a consequence of two distinct assumptions: $\mathrm{v}=0$ and $\boldsymbol{\sigma}_{s} \boldsymbol{n}_{1}=\boldsymbol{0}$ at $F_{1}=$ 0 . In the model by Berzi et al. [8-10] for a twolayer flow with a sediment-water mixture in the lower layer and clean water in the upper layer, $\boldsymbol{\sigma}_{s} \boldsymbol{n}_{1}$ $=\boldsymbol{O}$ is presumed at the layer interface and, as in Fraccarollo and Capart [3], a uniform $v$ along the avalanche depth is considered - this is different from (24). Here we use (24), which is based on (23), and deal with a solid volume fraction which slightly deviates from uniformity along the depth, except, eventually, near the layer interface. We introduce Boussinesq coefficients (see below) to account for these deviations, and let $v$ at the interface assume a value determined from experiments (Egashira et al. [30] used $v=0.05$, cf. Takahama et al. [7]).

(5) The free surface, say $\mathcal{S}_{2}$, is material for the upper fluid, since we do not consider any mass exchange at this surface. Therefore,

$$
\frac{\partial F_{2}}{\partial t}+\nabla F_{2} \cdot v_{2}=0, \text { at } F_{2}=0
$$

where $F_{2}\left(x_{1}, x_{2}, x_{3}, t\right)=0$ represents the equation of $\mathcal{S}_{2}$. Moreover, $\mathcal{S}_{2}$ is assumed traction-free:

$$
\boldsymbol{\sigma}_{2} \boldsymbol{n}_{2}=\boldsymbol{0} \text {, at } F_{2}=0 \text {, }
$$

where $\boldsymbol{n}_{2}$ is the unit normal vector to $\mathcal{S}_{2}$.

\subsection{Dimensionless Equations in Curvilinear Coordinates}

Using a typical length $L$ tangent to the topography, the gravitational acceleration $g$, and the densities $\tilde{\rho}_{s}$, $\tilde{\rho}_{f}$, we introduce non-dimensional fields as follows:

$$
\begin{aligned}
& \left(x_{1}, x_{2}, x_{3}, b\right)=L\left(\hat{x}_{1}, \hat{x}_{2}, \hat{x}_{3}, \hat{b}\right), \\
& \left(\boldsymbol{v}, \boldsymbol{u}, \boldsymbol{v}_{2}, t\right)=\sqrt{\operatorname{Lg}}\left(\hat{\boldsymbol{v}}, \hat{\boldsymbol{u}}, \hat{\boldsymbol{v}}_{2}, \hat{t} / L g\right), \\
& \boldsymbol{b}=g \hat{\boldsymbol{b}}, \stackrel{+}{\boldsymbol{m}}=\tilde{\rho}_{s} g \stackrel{\hat{\boldsymbol{m}}}{ }, \mathcal{M}_{i n t}=\tilde{\rho}_{f} \sqrt{L g} \mathcal{F}_{i n t}, \\
& \boldsymbol{\sigma}_{s}=\tilde{\rho}_{s} \operatorname{Lg} \hat{\boldsymbol{\sigma}}_{s},\left(\boldsymbol{\sigma}_{f}, \boldsymbol{\sigma}_{2}\right)=\tilde{\rho}_{f} \operatorname{Lg}\left(\hat{\boldsymbol{\sigma}}_{f}, \hat{\boldsymbol{\sigma}}_{2}\right) .
\end{aligned}
$$

Let $\left(\xi^{1}, \xi^{2}\right) \equiv \xi$ be length-dimensional surface parameters, and define non-dimensional $\hat{\xi}, \hat{\xi}$ by

$$
(\xi, \xi)=L(\hat{\xi}, \hat{\xi}) .
$$

Henceforth we shall omit the hat when dealing with dimensionless quantities. For instance, the nondimensional mass flux at the layer interface is

$$
\left.\mathcal{F}_{\text {int }} \equiv(1-\mathrm{v})\left(\boldsymbol{u} \cdot \boldsymbol{n}_{1}-\mathcal{U}_{1}\right)\right|_{F_{1}=0} .
$$

Thus, with the scalings (27), (28) and with

$$
c_{s f} \equiv \tilde{\rho}_{s} / \tilde{\rho}_{f},
$$

the modeling equations $(1) \sim(5)$ emerge as follows: 
$\left(1^{\prime}\right)$ in the lower layer, the mass and momentum balance equations for the solid constituent,

$$
\begin{aligned}
& \frac{\partial v}{\partial t}+\operatorname{div}(u v)=0 \\
& \frac{\partial v \boldsymbol{v}}{\partial t}+\operatorname{div}\left\{u \boldsymbol{v} \otimes \boldsymbol{v}-\sigma_{s}\right\}=v \boldsymbol{b}+\boldsymbol{m}
\end{aligned}
$$

and for the fluid constituent,

$$
\begin{aligned}
& \frac{\partial(1-v)}{\partial t}+\operatorname{div}\{(1-v) \boldsymbol{u}\}=0 \\
& \frac{\partial(1-v) \boldsymbol{u}}{\partial t}+\operatorname{div}\left\{(1-v) \boldsymbol{u} \otimes \boldsymbol{u}-\sigma_{f}\right\} \\
& =(1-v) \boldsymbol{b}-c_{s f} \boldsymbol{m}
\end{aligned}
$$

$\left(2^{\prime}\right)$ in the upper layer, the mass and momentum balance equations for the fluid,

$$
\begin{aligned}
& \operatorname{div} \boldsymbol{v}_{2}=0, \\
& \frac{\partial \boldsymbol{v}_{2}}{\partial t}+\operatorname{div}\left\{\boldsymbol{v}_{2} \otimes \boldsymbol{v}_{2}-\sigma_{2}\right\}=\boldsymbol{b} ;
\end{aligned}
$$

$\left(3^{\prime}\right)$ at the basal surface $x_{3}-b\left(x_{1}, x_{2}\right)=0$, the nonpenetration conditions

$$
\boldsymbol{v} \cdot \boldsymbol{n}=0, \boldsymbol{u} \cdot \boldsymbol{n}=0 ;
$$

(4') at the layer interface $F_{1}\left(x_{1}, x_{2}, x_{3}, t\right)=0$, the kinematic boundary condition

$$
\frac{\partial F_{1}}{\partial t}+\nabla F_{1} \cdot \boldsymbol{v}=0
$$

the jump of the fluid mass flow across $\mathcal{S}_{1}$,

$$
\boldsymbol{v}_{2} \cdot \boldsymbol{n}_{1}-\mathcal{U}_{1}=\mathcal{F}_{\text {int }},
$$

and the conditions on the tractions upon each side of $\mathcal{S}_{1}$,

$$
\begin{aligned}
& c_{s f} \boldsymbol{\sigma}_{2} \boldsymbol{n}_{1}=v \boldsymbol{\sigma}_{2} \boldsymbol{n}_{1}, \\
& \boldsymbol{\sigma}_{f} \boldsymbol{n}_{1}=(1-v) \sigma_{2} \boldsymbol{n}_{1}+\mathcal{F}_{\text {int }}\left(\boldsymbol{u}-\boldsymbol{v}_{2}\right) ;
\end{aligned}
$$

$\left(5^{\prime}\right)$ at the free surface $F_{2}\left(x_{1}, x_{2}, x_{3}, t\right)=0$, the kinematic condition and the traction-free condition

$$
\begin{aligned}
& \frac{\partial F_{2}}{\partial t}+\nabla F_{2} \cdot \boldsymbol{v}_{2}=0, \\
& \boldsymbol{\sigma}_{2} \boldsymbol{n}_{2}=\boldsymbol{0} .
\end{aligned}
$$

Relations (31) (40) involve the unknown fields $v, v$, $\boldsymbol{u}, \boldsymbol{v}_{2}, \boldsymbol{\sigma}_{s}, \boldsymbol{\sigma}_{f}, \boldsymbol{\sigma}_{2}, \quad \stackrel{\boldsymbol{m}}{,} F_{1}, F_{2}$. No constitutive assumptions have been made so far, except (23). The way in which we treat $(31) \sim(40)$ will be shown after the introduction of the topography-fitted curvilinear coordinates.

We use the representation of the stress tensors $\sigma_{s}, \sigma_{f}$, $\sigma_{2}$ in the form (14), i.e.,

$$
\boldsymbol{\sigma}_{s}=-p_{s} \boldsymbol{1}+\boldsymbol{\sigma}_{s}^{E}, \boldsymbol{\sigma}_{f}=-p_{f} \boldsymbol{1}+\boldsymbol{\sigma}_{f}^{E}, \boldsymbol{\sigma}_{2}=-p_{2} \boldsymbol{1}+\boldsymbol{\sigma}_{2}^{E} .
$$

With notations analogous to those in (15) we have

$$
\mathbf{T}_{s}=-p_{s} \mathbf{M}+\mathbf{P}_{s}, \mathbf{t}_{s}=\mathbf{p}_{s}, T_{s}^{33}=-p_{s}+P_{s}^{33},
$$

and similar relations for the fluid in both layers. Then, the terms in the momentum equations containing the Christoffel symbols accompanying the change of coordinates can be gathered in block-matrices of the form

$$
\Gamma(-p \mathbf{M}, \mathbf{0}) \equiv p\left\{\mathbf{B}^{-1} \frac{\partial \mathbf{B}}{\partial \xi^{\alpha}} \mathbf{M} \mathbf{e}_{\alpha}+\operatorname{tr}\left(\mathbf{W}(\mathbf{I}-\xi \mathbf{W})^{-1}\right) \mathbf{B}^{-1} \mathbf{s}\right\}
$$

$\Gamma(\mathbf{P}, \mathbf{p}) \equiv-\mathbf{B}^{-1} \frac{\partial \mathbf{B}}{\partial \xi^{\alpha}} \mathbf{P} \mathbf{e}_{\alpha}+2 \mathbf{B}^{-1} \mathbf{F W} \mathbf{p}+\Gamma(\mathbf{P}) \mathbf{B}^{-1} \mathbf{s}$,

$\Gamma(\mathbf{v}, \mathbf{v}) \equiv-\mathbf{B}^{-1} \frac{\partial \mathbf{B}}{\partial \xi^{\alpha}}(\mathbf{v} \otimes \mathbf{v}) \mathbf{e}_{\alpha}+2 \mathbf{v} \mathbf{B}^{-1} \mathbf{F W v}+\Gamma(\mathbf{v}) \mathbf{B}^{-1} \mathbf{s}$,

$\Gamma(\mathbf{P}) \equiv-\mathbf{B}^{T} \mathbf{F}^{-T} \mathbf{H} \cdot \mathbf{P}$,

$\Gamma(\mathbf{v}) \equiv-\mathbf{B}^{T} \mathbf{F}^{-T} \mathbf{H} \cdot(\mathbf{v} \otimes \mathbf{v})$,

with $\alpha=1,2$, see [25]; $p, \mathbf{v}$ are scalars, $\mathbf{p}, \mathbf{v}$ are 2column matrices, $\mathbf{P}$ is a $2 \times 2$ matrix, $\mathbf{e}_{1}, \mathbf{e}_{2}$ are defined in (1), and for $\mathbf{s}, \mathbf{F}, \mathbf{B}, \mathbf{M}, \mathbf{H}, \mathbf{W}$ see Sect. 2. The body force $\boldsymbol{b}$ is the gravitational force, so that its non- dimensional components $\mathbf{b}$ and $\mathbf{b}$ are

$$
\mathbf{b}=-c \mathbf{B}^{-1} \mathbf{s}, \quad \mathbf{b}=-c,
$$

see [25]. We have the following results:

(1") In the lower layer, following Luca et al. [27], the mass balance $(31)_{1}$ for the solid constituent reads

$$
\frac{\partial J u}{\partial t}+\operatorname{Div}\{J u \mathbf{v}\}+\frac{\partial}{\partial \xi}\{J u v\}=0
$$

and the momentum balance $(31)_{2}$ is separated into two scalar components parallel to the topography,

$$
\begin{aligned}
& \frac{\partial}{\partial t}\{J u \mathbf{v}\}+\operatorname{Div}\left\{J\left(u \mathbf{v} \otimes \mathbf{v}+p_{s} \mathbf{M}-\mathbf{P}_{s}\right)\right\} \\
& +\frac{\partial}{\partial \xi}\left\{J\left(u \mathbf{v}-\mathbf{p}_{s}\right)\right\}+J \Gamma\left(-p_{s} \mathbf{M}, \mathbf{0}\right) \\
& +J \Gamma\left(\mathbf{P}_{s}, \mathbf{p}_{s}\right)=J u \mathbf{b}+J v \Gamma(\mathbf{v}, \mathbf{v})+J \mathbf{m},
\end{aligned}
$$

and a scalar component normal to the topography,

$$
\begin{aligned}
& \frac{\partial}{\partial t}\{J u v\}+\operatorname{Div}\left\{J\left(u v \mathbf{v}-\mathbf{p}_{s}\right)\right\} \\
& +\frac{\partial}{\partial \xi}\left\{J\left(u v^{2}-P_{s}^{33}\right)\right\}+J \frac{\partial p_{s}}{\partial \xi} \\
& +J \Gamma\left(\mathbf{P}_{s}\right)=J u b+J v \Gamma(\mathbf{v})+J \mathbf{m},
\end{aligned}
$$

For the fluid constituent we have the mass balance,

$$
\begin{aligned}
& \frac{\partial}{\partial t}\{J(1-v)\}+\operatorname{Div}\{J(1-v) \mathbf{u}\} \\
& +\frac{\partial}{\partial \xi}\{J(1-v) u\}=0
\end{aligned}
$$

the momentum balance parallel to the topography, 


$$
\begin{aligned}
& \frac{\partial}{\partial t}\{J(1-v) \mathbf{u}\}+\operatorname{Div}\left\{J\left[(1-v) \mathbf{u} \otimes \mathbf{u}+p_{f} \mathbf{M}-\mathbf{P}_{f}\right]\right\} \\
& +\frac{\partial}{\partial \xi}\left\{J\left[(1-v) \mathbf{u} \mathbf{u}-\mathbf{p}_{f}\right]\right\}+J \Gamma\left(-p_{f} \mathbf{M}, 0\right)+J \Gamma\left(\mathbf{P}_{f}, \mathbf{p}_{f}\right) \\
& =J(1-v) \mathbf{b}+J(1-v) \Gamma(\mathbf{u}, \mathbf{u})-c_{s f} J \stackrel{+}{\mathbf{m}},
\end{aligned}
$$

and a momentum equation normal to the topography,

$$
\begin{aligned}
& \frac{\partial}{\partial t}\{J(1-v) \mathrm{u}\}+\operatorname{Div}\left\{J\left[(1-v) \mathrm{u} \mathbf{u}-\mathbf{p}_{f}\right]\right\} \\
& +\frac{\partial}{\partial \xi}\left\{J\left[(1-v) \mathrm{u}^{2}-P_{f}^{33}\right]\right\}+J \frac{\partial p_{f}}{\partial \xi}+J \Gamma\left(\mathbf{P}_{f}\right) \\
& =J(1-v) \mathrm{b}+J(1-v) \Gamma(\mathbf{u})-c_{s f} J \stackrel{+}{\mathrm{m}}
\end{aligned}
$$

$\left(2^{\prime \prime}\right)$ In the upper layer, Eq. (33), written in the curvilinear coordinates (9), appear as

$\operatorname{Div}\left\{J_{\mathbf{v}_{2}}\right\}+\frac{\partial}{\partial \xi}\left\{J_{\mathbf{v}_{2}}\right\}=0$,

$\frac{\partial}{\partial t}\left\{J \mathbf{v}_{2}\right\}+\operatorname{Div}\left\{J\left(\mathbf{v}_{2} \otimes \mathbf{v}_{2}+p_{2} \mathbf{M}-\mathbf{P}_{2}\right)\right\}$

$+\frac{\partial}{\partial \xi}\left\{J\left(\mathrm{v}_{2} \mathbf{v}_{2}-\mathbf{p}_{2}\right)\right\}+J \Gamma\left(-p_{2} \mathbf{M}, \mathbf{0}\right)$

$+J \Gamma\left(\mathbf{P}_{2}, \mathbf{p}_{2}\right)=J \mathbf{b}+J \Gamma\left(\mathbf{v}_{2}, \mathrm{v}_{2}\right)$,

$\frac{\partial}{\partial t}\left\{J_{\mathrm{v}_{2}}\right\}+\operatorname{Div}\left\{J\left(\mathrm{v}_{2} \mathbf{v}_{2}-\mathbf{p}_{2}\right)\right\}$

$+\frac{\partial}{\partial \xi}\left\{J \mathrm{v}_{2}\left(\mathrm{v}_{2}-P_{2}^{33}\right)\right\}+J \frac{\partial p_{2}}{\partial \xi}+J \Gamma\left(\mathbf{p}_{2}\right)$

$=J \mathrm{~b}+J \Gamma\left(\mathbf{v}_{2}\right)$.

(3") At the basal surface, $\xi=0$, conditions (34) of the tangency of the velocities emerge as

$$
\mathrm{v}=0, \mathrm{u}=0 \text { at } \xi=0 \text {. }
$$

(4") The equation for the layer interface is supposed to emerge as $F_{1}(\xi, \xi, t) \equiv \xi-h_{1}(\xi, t)=0$; thus,

$\boldsymbol{n}_{1}=\left.\frac{\nabla F_{1}}{\left\|\nabla F_{1}\right\|}\right|_{\xi=h_{1}}=\left.\frac{1}{\left\|\nabla F_{1}\right\|}\left(\boldsymbol{n}-\frac{\partial h_{1}}{\partial \xi^{\alpha}} \boldsymbol{g}^{\alpha}\right)\right|_{\xi=h_{1}}$,

where $\left\{\boldsymbol{g}^{1}, \boldsymbol{g}^{2}, \boldsymbol{g}^{3}=\boldsymbol{n}\right\}$ is the reciprocal basis of $\left\{\boldsymbol{g}_{1}\right.$, $\left.\boldsymbol{g}_{2}, \boldsymbol{g}_{3}\right\}$, and

$$
\mathcal{U}_{1}=\frac{1}{\left\|\nabla F_{1}\right\|_{\xi=h_{1}}} \frac{\partial h_{1}}{\partial t} .
$$

As a consequence, at $\xi=h_{1}$ we have:

(a) the kinematic boundary condition (35) reads

$$
\frac{\partial h_{1}}{\partial t}+\operatorname{Grad} h_{1} \cdot \mathbf{v}=\mathrm{v}
$$

(b) the jump relation (36) takes the form

$$
\frac{\partial h_{1}}{\partial t}+\operatorname{Grad} h_{1} \cdot \mathbf{v}_{2}=\mathrm{v}_{2}-\frac{1}{J} s_{\text {int }},
$$

where

$$
\begin{aligned}
& s_{\text {int }} \equiv\left\{J\left\|\nabla F_{1}\right\|\right\}_{\xi=h_{1}} \mathcal{F}_{\text {int }} \\
& =\left\{J(1-\mathrm{v})\left(\mathrm{u}-\frac{\partial h_{1}}{\partial t}-\operatorname{Grad} h_{1} \cdot \mathbf{u}\right)\right\}_{\xi=h_{1}} ;
\end{aligned}
$$

note the equivalence

$$
\mathcal{M}_{\text {int }}=0 \Leftrightarrow \mathcal{F}_{\text {int }}=0 \Leftrightarrow s_{\text {int }}=0 .
$$

(c) condition (37) emerges as

$$
\begin{aligned}
& c_{s f}\left\{\left(-p_{s} \mathbf{M}+\mathbf{P}_{s}\right) \operatorname{Grad} h_{1}-\mathbf{p}_{s}\right\} \\
& =v\left\{\left(-p_{2} \mathbf{M}+\mathbf{P}_{2}\right) \operatorname{Grad} h_{1}-\mathbf{p}_{2}\right\}, \\
& c_{s f}\left\{\mathbf{p}_{s} \cdot \operatorname{Grad} h_{1}+p_{s}-P_{s}^{33}\right\} \\
& =v\left\{\mathbf{p}_{2} \cdot \operatorname{Grad} h_{1}+p_{2}-P_{2}^{33}\right\},
\end{aligned}
$$

and (38) is written as

$$
\begin{aligned}
& \left(-p_{f} \mathbf{M}+\mathbf{P}_{f}\right) \mathrm{Grad} h_{1}-\mathbf{p}_{f} \\
= & (1-\mathrm{v})\left\{\left(-p_{2} \mathbf{M}+\mathbf{P}_{2}\right) \mathrm{Grad} h_{1}-\mathbf{p}_{2}\right\} \\
& -\frac{1}{J} s_{\text {int }}\left(\mathbf{u}-\mathbf{v}_{2}\right), \\
& \mathbf{p}_{f} \cdot \operatorname{Grad} h_{1}+p_{f}-P_{f}^{33} \\
= & (1-v)\left\{\mathbf{p}_{2} \cdot \operatorname{Grad} h_{1}+p_{2}-P_{2}^{33}\right\} \\
& -\frac{1}{J} s_{\text {int }}\left(\mathrm{u}-\mathrm{v}_{2}\right) .
\end{aligned}
$$

$\left(5^{\prime \prime}\right)$ The free surface equation $F_{2}\left(x_{1}, x_{2}, x_{3}, t\right)=0$ in the curvilinear coordinates (9) is assumed $F_{2}(\xi, \xi, t)$ $\equiv \xi-h_{2}(\xi, t)=0$. Thus, at $\xi=h_{2}$ (39) reads

$$
\frac{\partial h_{2}}{\partial t}+\operatorname{Grad} h_{2} \cdot \mathbf{v}_{2}=\mathrm{v}_{2}
$$

and the traction-free condition (40) can be written as

$$
\begin{aligned}
& \left(-p_{2} \mathbf{M}+\mathbf{P}_{2}\right) \operatorname{Grad} h_{2}-\mathbf{p}_{2}=\mathbf{0}, \\
& \mathbf{p}_{2} \cdot \operatorname{Grad} h_{2}+p_{2}-P_{2}^{33}=0 .
\end{aligned}
$$

\subsection{Depth-Averaging Procedure}

The equations, derived in Sect. 3.2, are next recast into a form which allows the derivation of the final equations involving depth-averages of the fields entering the flow model. Doing so we shall use the Leibniz formula

$$
\begin{aligned}
& \int_{f(\xi, t)}^{g(\xi, t)} \operatorname{Div} \Lambda d \xi=\operatorname{Div} \int_{f(\xi, t)}^{g(\xi, t)} \Lambda d \xi \\
& \quad+\Lambda(\xi, f(\xi, t)) \cdot \operatorname{Grad} f-\Lambda(\xi, g(\xi, t)) \cdot \operatorname{Grad} g,
\end{aligned}
$$

for a 2-column matrix $\Lambda=\mathbf{v}(\xi, \xi, t)$, and a square ma$\operatorname{trix} \Lambda=\mathbf{P}(\xi, \xi, t)$ of order 2 .

First we note that the normal velocity $\mathrm{V}$ of the solid constituent in the lower layer can be determined, with the boundary condition $(52)_{1}$, by integrating the mass balance equation (43) from 0 to $\xi \in\left(0, h_{1}\right)$ : 


$$
\mathrm{v}=-\frac{1}{J v} \int_{0}^{\xi}\left\{\frac{\partial J u}{\partial t}+\operatorname{Div} J u \mathbf{v}\right\} d \xi^{\prime} \text {. }
$$

Evaluating this at $\xi=h_{1}$, applying the Leibniz rule to the emerging expression and substituting the result into (54) yields

$$
\frac{\partial}{\partial t} \int_{0}^{h_{1}} J v d \xi+\operatorname{Div} \int_{0}^{h_{1}} J v \mathbf{v} d \xi=0 .
$$

Similarly, from the mass balance equation (46) for the fluid and the boundary condition $(52)_{2}$ we derive

$$
\begin{aligned}
\mathrm{u}= & -\frac{1}{J(1-v)} \int_{0}^{\xi}\left\{\frac{\partial}{\partial t}\{J(1-v)\}\right. \\
& +\operatorname{Div} J(1-v) \mathbf{u}\} d \xi^{\prime}
\end{aligned}
$$

In particular, this turns definition (56) of $s_{\text {int }}$ into

$$
S_{\text {int }} \equiv-\frac{\partial}{\partial t} \int_{0}^{h_{1}} J(1-\mathrm{v}) d \xi-\operatorname{Div} \int_{0}^{h_{1}} J(1-\mathrm{v}) \mathbf{u} d \xi
$$

Now, the velocity $\mathrm{v}_{2}$ of the fluid in the upper layer can be deduced by integrating mass balance (49) from $\xi \in\left(h_{1}, h_{2}\right)$ to $h_{2}$, and using (60). One obtains

$$
\begin{aligned}
\mathbf{v}_{2}= & \frac{\left.J\right|_{\xi=h_{2}}}{J}\left(\frac{\partial h_{2}}{\partial t}+\left.\operatorname{Grad} h_{2} \cdot \mathbf{v}_{2}\right|_{\xi=h_{2}}\right) \\
& +\frac{1}{J} \int_{\xi}^{h_{2}} \operatorname{Div} J \mathbf{v}_{2} d \xi^{\prime} .
\end{aligned}
$$

In particular, from (66) we can deduce $\left.\mathrm{v}_{2}\right|_{\xi=h_{1}}$, which, when inserted into (55), transforms (55) into

$$
\frac{\partial}{\partial t} \int_{h_{1}}^{h_{2}} J d \xi+\operatorname{Div} \int_{h_{1}}^{h_{2}} J \mathbf{v}_{2} d \xi=s_{i n t}
$$

We can refer to (63), (67) as the depth-integrated mass balance equations corresponding to the solid constituent in layer $\mathcal{E}_{1}$ and the fluid in layer $\mathcal{E}_{2}$. This is so, because (63), (67) can also be deduced by integrating (43) from 0 to $h_{1}$, and (49) from $h_{1}$ to $h_{2}$. Note that by integrating the mass balance Eq. (46) from 0 to $h_{1}$ one obtains the expression (65) of $s_{\text {int }}$. Thus, (65) can be conceived as the depth-integrated mass balance "equation" corresponding to the fluid constituent in layer $\mathcal{E}_{1}$. However, this interpretation is misleading, since (65) is not an equation: $s_{\text {int }}$ is the quantity defined in (56), and re-written as (65) by using the solution $\mathrm{u}$ of the BVP (46) and (52) $)_{2}$. Unlike (65), relations (63), (67) are equations, since they are obtained by introducing $\mathrm{v}, \mathrm{v}_{2}$ into the interface boundary conditions (54), (55). For immiscible layers we have the additional boundary condition $s_{\text {int }}=0$ at $\xi=h_{1}$, which yields the equation

$$
\frac{\partial}{\partial t} \int_{0}^{h_{1}} J(1-v) d \xi+\operatorname{Div} \int_{0}^{h_{1}} J(1-v) \mathbf{u} d \xi=0,
$$

which may be interpreted, without ambiguity, as the depth-integrated mass balance equation for the fluid in the lower layer.

Next we re-write the dynamic boundary conditions $(58)_{1},(59)_{1},(61)_{1}$. To this end we depth-average the tangential momentum balances (44), (47), (50), i.e., integrate (44), (47) from 0 to $h_{1}$, and (50) from $h_{1}$ to $h_{2}$. After straightforward calculations using the Leibniz rule, the expressions

$$
\begin{aligned}
& \left\{\left(-p_{s, f} \mathbf{M}+\mathbf{P}_{s, f}\right) \operatorname{Grad} h_{1}-\mathbf{p}_{s, f}\right\}_{\xi=h_{1}}, \\
& \left\{\left(-p_{2} \mathbf{M}+\mathbf{P}_{2}\right) \operatorname{Grad} h_{2}-\mathbf{p}_{2}\right\}_{\xi=h_{2}},
\end{aligned}
$$

can be identified in the emerging formulae, which can be combined with $(58)_{1},(59)_{1},(61)_{1}$. The result are the following depth-integrated momentum equations:

$$
\begin{aligned}
& \frac{\partial}{\partial t} \int_{0}^{h_{1}} J v \mathbf{v} d \xi+\operatorname{Div} \int_{0}^{h_{1}} J\left\{v \mathbf{v} \otimes \mathbf{v}+p_{s} \mathbf{M}-\mathbf{P}_{s}\right\} d \xi \\
& +\frac{1}{c_{s f}}\left\{J v\left[\left(-p_{2} \mathbf{M}+\mathbf{P}_{2}\right) \operatorname{Grad} h_{1}-\mathbf{p}_{2}\right]\right\}_{\xi=h_{1}} \\
& +\left.\left(J \mathbf{p}_{s}\right)\right|_{\xi=0}+\int_{0}^{h_{1}} J\left\{\Gamma\left(-p_{s} \mathbf{M}, \mathbf{0}\right)+\Gamma\left(\mathbf{P}_{s}, \mathbf{p}_{s}\right)\right\} d \xi \\
& =\int_{0}^{h_{1}} J v \mathbf{b} d \xi+\int_{0}^{h_{1}} J v \Gamma(\mathbf{v}, \mathbf{v}) d \xi+\int_{0}^{h_{1}} J \mathbf{m} d \xi \\
& \frac{\partial}{\partial t} \int_{0}^{h_{1}} J(1-v) \mathbf{u} d \xi+\operatorname{Div} \int_{0}^{h_{1}} J\left\{(1-v) \mathbf{u} \otimes \mathbf{u}+p_{f} \mathbf{M}-\mathbf{P}_{f}\right\} d \xi \\
& +\left\{J(1-v)\left[\left(-p_{2} \mathbf{M}+\mathbf{P}_{2}\right) \operatorname{Grad} h_{1}-\mathbf{p}_{2}\right]\right\}_{\xi=h_{1}} \\
& +\left.\left(J \mathbf{p}_{f}\right)\right|_{\xi=0}+\int_{0}^{h_{1}} J\left\{\Gamma\left(-p_{f} \mathbf{M}, \mathbf{0}\right)+\Gamma\left(\mathbf{P}_{f}, \mathbf{p}_{f}\right)\right\} d \xi \\
& =\int_{0}^{h_{1}} J(1-v) \mathbf{b} d \xi+\int_{0}^{h_{1}} J(1-v) \Gamma(\mathbf{u}, \mathbf{u}) d \xi \\
& \quad-c_{s f} \int_{0}^{h_{1}} J \stackrel{+}{\mathbf{m}} d \xi-\left.s_{i n t} \mathbf{v}_{2}\right|_{\xi=h_{1}},
\end{aligned}
$$

and

$$
\begin{aligned}
& \frac{\partial}{\partial t} \int_{h_{1}}^{h_{2}} J \mathbf{v}_{2} d \xi+\operatorname{Div} \int_{h_{1}}^{h_{2}} J\left\{\mathbf{v}_{2} \otimes \mathbf{v}_{2}+p_{2} \mathbf{M}-\mathbf{P}_{2}\right\} d \xi \\
& -\left\{J\left[\left(-p_{2} \mathbf{M}+\mathbf{P}_{2}\right) \operatorname{Grad} h_{1}-\mathbf{p}_{2}\right]\right\}_{\xi=h_{1}} \\
& +\int_{h_{1}}^{h_{2}} J\left\{\Gamma\left(-p_{2} \mathbf{M}, \mathbf{0}\right)+\Gamma\left(\mathbf{P}_{2}, \mathbf{p}_{2}\right)\right\} d \xi \\
& =\int_{h_{1}}^{h_{2}} J \mathbf{b} d \xi+\int_{h_{1}}^{h_{2}} J \Gamma\left(\mathbf{v}_{2}, \mathbf{v}_{2}\right) d \xi+\left.s_{\text {int }} \mathbf{v}_{2}\right|_{\xi=h_{1}},
\end{aligned}
$$

for the solid in $\mathcal{E}_{1}$, the fluid in $\mathcal{E}_{1}$ and the fluid in $\mathcal{E}_{2}$. We summarize the modeling equations of $\left(1^{\prime \prime}\right) \sim\left(5^{\prime \prime}\right)$ in Table 2 together with their physical meaning. In these equations $\mathrm{v}, \mathrm{u}, \mathrm{v}_{2}$ are given by (62), (64), (66).

To cope with the complexity of these equations we follow the so-called depth-averaging procedure, i.e., we ignore the local tangential momentum balance equations (44), (47), (50), and exploit the remaining system of equations. More exactly, letting $f$ be a function defined in the lower layer and $g$ in the upper layer, we introduce the depth-averages $\bar{f}$ and $\bar{g}$ by the expressions 
Table 2 Summary of model equations

\begin{tabular}{|l|c|}
\hline \multicolumn{1}{|c|}{ Physical statement } & Eq. No. \\
\hline $\begin{array}{l}\text { Local tangential/normal balance of solid } \\
\text { momentum in } \mathcal{E}_{1}\end{array}$ & $(44),(45)$ \\
\hline $\begin{array}{l}\text { Local tangential/normal balance of fluid } \\
\text { momentum in } \mathcal{E}_{1}\end{array}$ & $(47),(48)$ \\
\hline $\begin{array}{l}\text { Local tangential/normal balance of fluid } \\
\text { momentum in } \mathcal{E}_{2}\end{array}$ & $(50),(51)$ \\
\hline Depth-integrated mass balance of the solid in $\mathcal{E}_{1}$ & $(63)$ \\
\hline Depth-integrated mass balance of the fluid in $\mathcal{E}_{2}$ & $(67)$ \\
\hline Expression of $s_{\text {int }}$ & $(64)$ \\
\hline $\begin{array}{l}\text { Depth-integrated tangential momentum balance } \\
\text { of the solid in } \mathcal{E}_{1}\end{array}$ & $(68)$ \\
\hline $\begin{array}{l}\text { Depth-integrated tangential momentum balance } \\
\text { of the fluid in } \mathcal{E}_{1}\end{array}$ & $(69)$ \\
\hline $\begin{array}{l}\text { Depth-integrated tangential momentum balance } \\
\text { of the fluid in } \mathcal{E}_{2}\end{array}$ & $(70)$ \\
\hline $\begin{array}{l}\text { Normal traction condition at } \mathcal{S}_{1} \text { for the solid } \\
\text { constituent }\end{array}$ & $(58)_{2}$ \\
\hline $\begin{array}{l}\text { Normal traction condition at } \mathcal{S}_{1} \text { for the fluid } \\
\text { constituent }\end{array}$ & $(59)_{2}$ \\
\hline $\begin{array}{l}\text { Normal traction condition at for } \mathcal{S}_{2} \text { the fluid } \\
\text { constituent }\end{array}$ & $(61)_{2}$ \\
\hline
\end{tabular}

$\bar{f} \equiv \frac{1}{h_{1}} \int_{0}^{h_{1}} f(\xi, \xi, t) d \xi, \quad \bar{g} \equiv \frac{1}{h} \int_{h_{1}}^{h_{2}} g(\xi, \xi, t) d \xi$,

where $h \equiv h_{2}-h_{1}$. Then, we (i) make ordering approximations justified by the shallowness of the avalanching mass, (ii) determine the pressures $p_{s}, p_{f}$ and $p_{2}$ from the normal momentum balances together with corresponding boundary conditions and constitutive assumptions, and (iii) transform the depth-averaged mass and momentum balances into equations for the determination of the basic scalar fields $\bar{v}, \overline{\mathbf{v}}, \overline{\mathbf{u}}, \overline{\mathbf{v}}_{\mathbf{2}}$, $h_{1}, h_{2}$.

\subsection{Ordering Approximations}

For the derivation of the final modeling equations we use the same scaling approximations as in [27,5]. We express the approximations in terms of an aspect ratio $\epsilon$ $\equiv H / L<<1$, where $H$ is a typical thickness normal to the topography and $L$ is the typical length-scale tangent to the topography. A constant $\gamma \in(0,1)$ is also used.

(a) Geometric approximations: Both layers are thin, in the sense that $h_{2}=O(\epsilon)$, which implies $\xi=O(\epsilon)$ for $\xi \in\left(0, h_{2}\right)$, in particular for $\xi=h_{1}$.

(b) Kinematic approximations: The tangential velocities $\mathbf{v}, \mathbf{u}, \mathbf{v}_{\mathbf{2}}$ are $O(1)$, the solid velocity satisfies

$$
\begin{aligned}
& \int_{0}^{h_{1}} \xi \mathbf{v} d \xi=\frac{1}{2} h_{1}^{2} m_{1}^{(s)} \overline{\mathbf{v}}+O\left(\epsilon^{2+\gamma}\right), \\
& \int_{0}^{h_{1}} \mathbf{v} \otimes \mathbf{v} d \xi=h_{1} m_{2}^{(s)} \overline{\mathbf{v}} \otimes \overline{\mathbf{v}}+O\left(\epsilon^{2+\gamma}\right), \\
& \int_{0}^{h_{1}} \xi \mathbf{v} \otimes \mathbf{v} d \xi=\frac{1}{2} h_{1}^{2} m_{3}^{(s)} \overline{\mathbf{v}} \otimes \overline{\mathbf{v}}+O\left(\epsilon^{2+\gamma}\right), \\
& \int_{0}^{h_{1}} \mathbf{v} \mathbf{v} d \xi=\frac{1}{2} h_{1}^{2} \beta_{s} \overline{\mathbf{v}}+O\left(\epsilon^{2+\gamma}\right),
\end{aligned}
$$

and similar relations hold with scalar coefficients $m_{1}^{(f)}, m_{2}^{(f)}, m_{3}^{(f)}, \beta_{f}$ for the pore fluid velocity. In the upper layer we assume

$$
\begin{aligned}
& \int_{h_{1}}^{h_{2}} \xi \mathbf{v}_{2} d \xi=\frac{1}{2} h\left(h_{1}+h_{2}\right) m_{1}^{(2)} \overline{\mathbf{v}}_{2}+O\left(\epsilon^{2+\gamma}\right), \\
& \int_{h_{1}}^{h_{2}} \mathbf{v}_{2} \otimes \mathbf{v}_{2} d \xi=h m_{2}^{(2)} \overline{\mathbf{v}}_{2} \otimes \overline{\mathbf{v}}_{2}+O\left(\epsilon^{2+\gamma}\right), \\
& \int_{h_{1}}^{h_{2}} \xi \mathbf{v}_{2} \otimes \mathbf{v}_{2} d \xi=\frac{1}{2} h\left(h_{1}+h_{2}\right) m_{3}^{(2)} \overline{\mathbf{v}}_{2} \otimes \overline{\mathbf{v}}_{2}+O\left(\epsilon^{2+\gamma}\right), \\
& \int_{h_{1}}^{h_{2}} \mathbf{v}_{2} \mathbf{v}_{2} d \xi=h\left(\frac{\partial h_{2}}{\partial t}+\operatorname{Grad} h_{2} \cdot \overline{\mathbf{v}}_{2}\right) \overline{\mathbf{v}}_{2} \\
& \quad+\frac{1}{2} h^{2} \beta_{2} \overline{\mathbf{v}}_{2}+O\left(\epsilon^{2+\gamma}\right),
\end{aligned}
$$

owing to (66). Here, $h \equiv h_{2}-h_{1}$. The correction factors (or Boussinesq coefficients) $m_{1}^{(s)}$ to $\beta_{2}$ are supposed to be scalar functions of $\xi, t$ of order $O(1)$.

(c) Configurational-kinematic approximations: The solid volume fraction $v$ and the tangential velocities $\mathbf{v}, \mathbf{u}$ are assumed to satisfy the relations

$$
v=\bar{v}+O(\epsilon), \quad \bar{v}=O(1),
$$

and

$$
\begin{aligned}
& \int_{0}^{h_{1}} v \mathbf{v} d \xi=h_{1} n_{1}^{(s)} \bar{v} \overline{\mathbf{v}}+O\left(\epsilon^{2+\gamma}\right), \\
& \int_{0}^{h_{1}} v \mathbf{v} \otimes \mathbf{v} d \xi=h_{1} n_{2}^{(s)} \overline{\mathrm{v}} \overline{\mathbf{v}} \otimes \overline{\mathbf{v}}+O\left(\epsilon^{2+\gamma}\right), \\
& \int_{0}^{h_{1}} \mathrm{v} \mathbf{u} d \xi=h_{1} n_{1}^{(f)} \overline{\mathrm{v}} \overline{\mathbf{u}}+O\left(\epsilon^{2+\gamma}\right), \\
& \int_{0}^{h_{1}} \mathrm{v} \mathbf{u} \otimes \mathbf{u} d \xi=h_{1} n_{2}^{(f)} \overline{\mathrm{v}} \overline{\mathbf{u}} \otimes \overline{\mathbf{u}}+O\left(\epsilon^{2+\gamma}\right),
\end{aligned}
$$

where the scalar coefficients $n_{1}^{(s)}$ to $n_{2}^{(f)}$ are functions of $\xi$, $t$ of order $O(1)$.

(d) Dynamic stress approximations: It is conjectured that the stress tensors $\sigma_{s}, \sigma_{f}, \sigma_{2}$ satisfy the order relations

$$
\begin{aligned}
& p_{s, f, 2}=O(\epsilon), \mathbf{P}_{s, f, 2}=O(\epsilon), \\
& \mathbf{p}_{s, f, 2}=O\left(\epsilon^{\gamma}\right), P_{s, f, 2}^{33}=O\left(\epsilon^{1+\gamma}\right) .
\end{aligned}
$$

Accordingly, Fig. 2, the shear stresses on planes perpendicular to the base and pointing upwards are the strongest, since this type of shearing is large, Fig. 2(a). The constraint pressure is essentially hydrostatic and therefore of $O(\epsilon)$, and of equal order are the shear stresses parallel to the base, Fig. 2(b). Finally, the normal stress beyond the hydrostatic pressure, orthogonal to the base and responsible for the normal stress effects is the smallest and of 


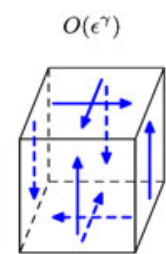

$\mathbf{p}_{s, f, 2}$

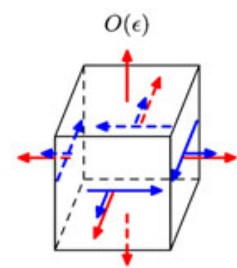

$\mathbf{P}_{s, f, 2}, p_{s, f, 2}$

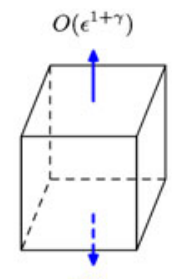

$P_{s, f, 2}^{33}$ (b) (c)

Fig. 2 Material differential element with surfaces parallel to $\xi^{1}, \xi^{2}$ and perpendicular to $\mathcal{S}$. Plotted are the stress components (dashed on invisible faces) of (76)

$O\left(\epsilon^{1+\gamma}\right)$, Fig. 2(c). For further details on assumptions $(\mathrm{a}) \sim(\mathrm{d})$ see $[27,5]$. Here we additionally mention that, (i) instead of specifying the tangential velocity profile, we have introduced Boussinesq coefficients which account for deviations of the tangential velocities from the plug-flow profile ${ }^{2}$, (ii) assumption (73) $)_{4}$ is justified by formula (66) for the normal velocity $v_{2}$ in the same way as it has been done in [5] for a similar situation, and (iii) postulating (74), e.g., a profile of the solid volume fraction, linear in $\xi$, is envisaged.

According to (62), (64), (66), since $\mathbf{v}, \mathbf{u}, \mathbf{v}_{2}, v, J$ are $O(1), \xi=O(\epsilon)$, the velocities $\mathrm{v}, \mathrm{u}, \mathrm{v}_{2}$ appear as

$$
\mathrm{v}=O(\epsilon), \mathrm{u}=O(\epsilon), \quad \mathrm{v}_{2}=O(\epsilon),
$$

which will henceforth be used at some occasions. The following estimations are also needed:

$$
\begin{aligned}
& J=J_{0}(1-2 \Omega \xi)+O\left(\epsilon^{2}\right),\left.\quad J_{0} \equiv J\right|_{\xi=0}=\frac{1}{c} \operatorname{det} \mathbf{F}, \\
& J \mathbf{v}=J_{0}\{\mathbf{v}-2 \Omega(\xi \mathbf{v})\}+O\left(\epsilon^{2}\right), \\
& J \mathbf{v} \otimes \mathbf{v}=J_{0}\{(\mathbf{v} \otimes \mathbf{v})-2 \Omega(\xi \mathbf{v} \otimes \mathbf{v})\}+O\left(\epsilon^{2}\right), \\
& J(p \mathbf{M}-\mathbf{P})=J_{0}\left(p^{*} \mathbf{M}_{0}-\mathbf{P}\right)+O\left(\epsilon^{1+\gamma}\right), \\
& J \Gamma(-p \mathbf{M}, \mathbf{0}) \\
& =J_{0} p^{*}\left\{\mathbf{F}^{-1} \frac{\partial \mathbf{F}}{\partial \xi^{\alpha}} \mathbf{M}_{0} e_{\alpha}+2 \Omega \mathbf{F}^{-1} \mathbf{s}\right\}+O\left(\epsilon^{1+\gamma}\right), \\
& J \Gamma(\mathbf{P}, \mathbf{p})=-J_{0}\left\{\mathbf{F}^{-1} \frac{\partial \mathbf{F}}{\partial \xi^{\alpha}} \mathbf{P} \mathbf{e}_{\alpha}-2 \mathbf{W} \mathbf{p}\right. \\
& \left.+(\mathbf{H} \cdot \mathbf{P}) \mathbf{F}^{-1} \mathbf{s}\right\}+O\left(\epsilon^{1+\gamma}\right), \\
& J \Gamma(\mathbf{v}, \mathbf{v})=-J_{0} \mathbf{F}^{-1} \frac{\partial \mathbf{F}}{\partial \xi^{\alpha}}\{(\mathbf{v} \otimes \mathbf{v})-2 \Omega(\xi \mathbf{v} \otimes \mathbf{v})\} \mathbf{e}_{\alpha} \\
& +J_{0} \mathbf{F}^{-1} \frac{\partial}{\partial \xi^{\alpha}}\left(\mathbf{F} \mathbf{W} \mathbf{F}^{-1}\right) \mathbf{F}(\xi \mathbf{v} \otimes \mathbf{v}) \mathbf{e}_{\alpha} \\
& +2 J_{0} \mathbf{W}(\mathbf{v} \mathbf{v})-J_{0}\{\mathbf{H} \cdot(\mathbf{v} \otimes \mathbf{v})-2 \Omega \mathbf{H} \cdot(\xi \mathbf{v} \otimes \mathbf{v})\} \\
& \left.-\mathbf{W}^{T} \mathbf{H} \cdot(\xi \mathbf{v} \otimes \mathbf{v})\right\} \mathbf{F}^{-1} \mathbf{s} \\
& -J_{0}(\mathbf{H} \cdot(\xi \mathbf{v} \otimes \mathbf{v})) \mathbf{W} \mathbf{F}^{-1} \mathbf{s}+O\left(\epsilon^{2}\right), \\
& J \mathbf{b}=-J_{0} c(1-2 \Omega \xi) \mathbf{F}^{-1} \mathbf{s}-J_{0} c \xi \mathbf{W} \mathbf{F}^{-1} \mathbf{s}+O\left(\epsilon^{2}\right) ;
\end{aligned}
$$

\footnotetext{
2 Larcan et al. [1] pointed out that their experiments never evidenced a plug flow of the avalanching mass.
}

they hold for a two-column $\mathbf{v}=O(1)$, a scalar $\mathrm{v}=O(\epsilon)$, a $2 \times 2$ matrix $\mathbf{P}=O(\epsilon)$, a two-column $\mathbf{p}=O\left(\epsilon^{\gamma}\right)$, a sca$\operatorname{lar} p=p^{\star}+O\left(\epsilon^{1+\gamma}\right), p^{\star}=O(\epsilon)$, and $\mathbf{b}$ from (42).

\subsection{Shallow Flow Equations}

Next we shall use the assumed ordering approximations to deduce the equations that model the shallow flow of the two-layer system.

In order to determine the pressures $p_{s}, p_{f}$ from the normal momentum balance Eqs. (45), (48) with the aid of the boundary conditions $(58)_{2},(59)_{2}$, we need a constitutive law for the interaction force $\boldsymbol{m}$. In an ad-hoc manner, for $\stackrel{+}{\boldsymbol{m}}$ we take the (dimensional) law

$$
\stackrel{+}{\boldsymbol{m}}=\left\{\left(1-c_{s}\right) \tilde{p}_{s}+c_{s} \tilde{p}_{f}\right\} \nabla v+c_{D}(\boldsymbol{u}-\boldsymbol{v}),
$$

see Schneider and Hutter [33], where the true pressures $\tilde{p}_{s}, \quad \tilde{p}_{f}$ are defined by the equalities

$$
p_{s}=v \tilde{p}_{s}, \quad p_{f}=(1-v) \tilde{p}_{f},
$$

$c_{s}$ represents the solid mass fraction,

$$
c_{s} \equiv \frac{\rho_{s}}{\rho_{s}+\rho_{f}}=\frac{c_{s f} \mathrm{v}}{c_{s f} \mathrm{v}+1-\mathrm{v}},
$$

and the drag coefficient $c_{D}$ is supposed to be constant. The non-dimensional components of $\stackrel{+}{\boldsymbol{m}}$ then read

$$
\begin{aligned}
& \stackrel{+}{\mathbf{m}}=\left\{\left(1-c_{s}\right) \tilde{p}_{s}+\frac{c_{s}}{c_{s f}} \tilde{p}_{f}\right\} \mathbf{M G r a d} \mathrm{u}+c_{D}(\mathbf{u}-\mathbf{v}), \\
& +\stackrel{\mathrm{m}}{=}=\left\{\left(1-c_{s}\right) \tilde{p}_{s}+\frac{c_{s}}{c_{s f}} \tilde{p}_{f}\right\} \frac{\partial \mathrm{v}}{\partial \xi}+c_{D}(\mathrm{u}-\mathrm{v}),
\end{aligned}
$$

where the non-dimensional drag coefficient satisfies

$$
c_{D \text { non-dim }}=\frac{1}{\tilde{\rho}_{s}} \sqrt{\frac{L}{g}} c_{D \operatorname{dim}} .
$$

With $\stackrel{+}{\boldsymbol{m}}$ at hand we can now determine the pressures $p_{s}, p_{f}$. Thus, appealing to the ordering approximations, the boundary conditions $(58)_{2},(59)_{2}$ read

$$
\tilde{p}_{s}=\frac{1}{c_{s f}} p_{2}+O\left(\epsilon^{1+\gamma}\right), \quad \tilde{p}_{f}=p_{2}+O\left(\epsilon^{1+\gamma}\right)
$$

at $\xi=h_{1}$, and therefore we need first to determine $p_{2}$. Equations in the upper layer coincide with those in [5], if therein the basal topography is fixed, and hence we can invoke the results of the mentioned paper:

$$
\begin{aligned}
& \bar{p}_{2}=\frac{1}{2} h\left(c+a_{2} m_{3}^{(2)}\right)+h_{1} a_{2}\left(m_{3}^{(2)}-m_{2}^{(2)}\right)+O\left(\epsilon^{1+\gamma}\right), \\
& \left.p_{2}\right|_{\xi=h_{1}}=h\left(c+a_{2} m_{2}^{(2)}\right)+O\left(\epsilon^{1+\gamma}\right), \quad a_{2} \equiv \mathbf{H} \overline{\mathbf{v}}_{2} \cdot \overline{\mathbf{v}}_{2} .
\end{aligned}
$$

The determination of $\tilde{p}_{s}, \quad \tilde{p}_{f}$ can now be done in the same manner as in [27], by using (79) and $(80)_{2}$. Thus, 


$$
\begin{aligned}
\bar{p}_{s}= & \overline{\mathrm{v}}\left\{\frac{1}{2} h_{1}\left(c+a_{s} m_{3}^{(s)}\right)\right. \\
& \left.+\frac{1}{c_{s f}} h\left(c+a_{2} m_{2}^{(2)}\right)\right\}+O\left(\epsilon^{1+\gamma}\right), \\
\bar{p}_{f}= & (1-\overline{\mathrm{v}})\left\{\frac{1}{2} h_{1}\left(c+a_{f} m_{3}^{(f)}\right)\right. \\
& \left.+h\left(c+a_{2} m_{2}^{(2)}\right)\right\}+O\left(\epsilon^{1+\gamma}\right),
\end{aligned}
$$

where $a_{s} \equiv \mathbf{H} \overline{\mathbf{v}} \cdot \overline{\mathbf{v}}$ and $a_{f} \equiv \mathbf{H} \overline{\mathbf{u}} \cdot \overline{\mathbf{u}}$, and the basal solid pressure is obtained as

$$
\begin{aligned}
\left.p_{s}\right|_{\xi=0}= & \bar{v}\left\{h_{1}\left(c+a_{s} m_{2}^{(s)}\right)\right. \\
& \left.+\frac{1}{c_{s f}} h\left(c+a_{2} m_{2}^{(2)}\right)\right\}+O\left(\epsilon^{1+\gamma}\right) .
\end{aligned}
$$

Now, we focus on the depth-integrated mass and tangential momentum balance Eqs. (63), (67), (68) (70). We follow the same approach as in [27,5] with the aid of (77). Here is the result:

Except for negligible terms of order $O\left(\epsilon^{2+\gamma}\right)$, Eqs. (63), (67), (68) (70) appear as follows:

- The depth-integrated mass and tangential momentum balances (63), (68) for the solid in $\mathcal{E}_{1}$ turn into

$\mathcal{M}_{s}=0$,

$\mathcal{L}_{s}=\boldsymbol{R}_{s}$

$-\frac{1}{c_{s f}} \mathbf{F}\left\{J \cup\left[\left(-p_{2} \mathbf{M}+\mathbf{P}_{2}\right) \operatorname{Grad} h_{1}-\mathbf{p}_{2}\right]\right\}_{\xi=h_{1}}$,

where

$$
\begin{aligned}
\mathcal{M}_{s} \equiv & \frac{\partial}{\partial t}\left\{J_{0} h_{1}\left(1-\Omega h_{1}\right) \overline{\mathrm{v}}\right\} \\
& +\operatorname{Div}\left\{J_{0} h_{1}\left(n_{1}^{(s)}-\Omega h_{1} m_{1}^{(s)}\right) \overline{\mathrm{v}} \overline{\mathbf{v}}\right\} \\
\mathcal{L}_{s} \equiv & \frac{\partial}{\partial t}\left\{J_{0} h_{1}\left(n_{1}^{(s)}-\Omega h_{1} m_{1}^{(s)}\right) \overline{\mathrm{v}} \mathbf{F} \overline{\mathbf{v}}\right\} \\
& +\operatorname{Div}\left\{J _ { 0 } h _ { 1 } \mathbf { F } \left[\left(n_{2}^{(s)}-\Omega h_{1} m_{3}^{(s)}\right) \overline{\mathrm{v}} \overline{\mathbf{v}} \otimes \overline{\mathbf{v}}\right.\right. \\
& \left.\left.+\bar{p}_{s} \mathbf{M}_{0}-\overline{\mathbf{P}}_{s}\right]\right\}+2 J_{0} h_{1} \mathbf{F} \mathbf{W} \overline{\mathbf{p}}_{s}-J_{0} h_{1}\left(\mathbf{H} \cdot \overline{\mathbf{P}}_{s}\right) \mathbf{s}, \\
\boldsymbol{R}_{s} \equiv & -\left.J_{0} \mathbf{F} \mathbf{p}_{s}\right|_{\xi=0}-J_{0} h_{1}\left\{2 \Omega \bar{p}_{s}+c\left(1-\Omega h_{1}\right) \overline{\mathrm{v}}\right. \\
& \left.+\left(n_{2}^{(s)}-\Omega h_{1} m_{3}^{(s)}\right) a_{s} \overline{\mathrm{v}}-\frac{1}{2} h_{1} \tilde{a}_{s} m_{3}^{(s)} \overline{\mathrm{v}}\right\} \mathbf{s} \\
& -\frac{1}{2} J_{0} h_{1}^{2}\left(c+a_{s} m_{3}^{(s)}\right) \overline{\mathrm{v}} \mathbf{F} \mathbf{W} \mathbf{F}^{-1} \mathbf{s} \\
& +\frac{1}{2} J_{0} h_{1}^{2} m_{3}^{(s)} \overline{\mathrm{v}} \frac{\partial}{\partial \xi^{\alpha}}\left(\mathbf{F W} \mathbf{F}^{-1}\right) \mathbf{F}(\overline{\mathbf{v}} \otimes \overline{\mathbf{v}}) \mathbf{e}_{\alpha} \\
& +J_{0} h_{1}^{2} \beta_{s} \overline{\mathrm{v}} \mathbf{F W} \overline{\mathbf{v}}+\frac{J_{0} h_{1}^{2}}{2\left(c_{s f} \overline{\mathrm{v}}+1-\overline{\mathrm{v}}\right)} \\
& \times\left\{c+a_{s} m_{3}^{(s)}(1-\overline{\mathrm{v}})+a_{f} m_{3}^{(f)} \overline{\mathrm{v}}\right\} \mathbf{F} \mathbf{M}_{0} \mathrm{Grad} \overline{\mathrm{v}} \\
& +\frac{1}{c_{s f}} J_{0} h_{1} h\left(c+a_{2} m_{2}^{(2)}\right) \mathbf{F} \mathbf{M}_{0} \mathrm{Grad} \overline{\mathrm{v}} \\
& +c_{D} J_{0} h_{1}\left\{\left(1-\Omega h_{1} m_{1}^{(f)}\right) \mathbf{F} \overline{\mathbf{u}}-\left(1-\Omega h_{1} m_{1}^{(s)}\right) \mathbf{F} \overline{\mathbf{v}}\right\}
\end{aligned}
$$

with $\quad \tilde{a}_{s} \equiv \mathbf{H} \overline{\mathbf{v}} \cdot \mathbf{W} \overline{\mathbf{v}}$, and $\bar{p}_{s}$ as given by $(81)_{1}$.

- The depth-integrated tangential momentum balance (69) for the fluid in $\mathcal{E}_{1}$ reads

$$
\begin{aligned}
\mathcal{L}_{f}= & \boldsymbol{R}_{f}-\mathbf{F}\left\{J(1-v)\left[\left(-p_{2} \mathbf{M}+\mathbf{P}_{2}\right) \operatorname{Grad} h_{1}-\mathbf{p}_{2}\right]\right\}_{\xi=h_{1}} \\
& -\left.s_{\text {int }} \mathbf{F} \mathbf{v}_{2}\right|_{\xi=h_{1}},
\end{aligned}
$$

where

$$
\begin{aligned}
& \mathcal{L}_{f} \equiv \frac{\partial}{\partial t}\left\{J_{0} h_{1}\left(1-n_{1}^{(f)} \bar{v}-\Omega h_{1} m_{1}^{(f)}(1-\bar{v})\right) \mathbf{F} \overline{\mathbf{u}}\right\} \\
& +\operatorname{Div}\left\{J _ { 0 } h _ { 1 } \mathbf { F } \left[\left(m_{2}^{(f)}-n_{2}^{(f)} \bar{v}-\Omega h_{1} m_{3}^{(f)}(1-\bar{v})\right) \overline{\mathbf{u}} \otimes \overline{\mathbf{u}}\right.\right. \\
& \left.\left.+\bar{p}_{f} \mathbf{M}_{0}-\overline{\mathbf{P}}_{f}\right]\right\}+2 J_{0} h_{1} \mathbf{F W} \overline{\mathbf{p}}_{f}-J_{0} h_{1}\left(\mathbf{H} \cdot \overline{\mathbf{P}}_{f}\right) \mathbf{s}, \\
& \boldsymbol{R}_{f} \equiv-\left.J_{0} \mathbf{F} \mathbf{p}_{f}\right|_{\xi=0}-J_{0} h_{1} \\
& \times\left\{2 \Omega \bar{p}_{f}+c\left(1-\Omega h_{1}\right)(1-\overline{\mathrm{v}})+a_{f}\left(m_{2}^{(f)}-n_{2}^{(f)} \overline{\mathrm{v}}\right)\right. \\
& \left.-\Omega h_{1} a_{f} m_{3}^{(f)}(1-\overline{\mathrm{v}})-\frac{1}{2} h_{1} \tilde{a}_{f} m_{3}^{(f)}(1-\overline{\mathrm{v}})\right\} \mathbf{s} \\
& -\frac{1}{2} J_{0} h_{1}^{2}\left(c+a_{f} m_{3}^{(s)}\right)(1-\bar{v}) \mathbf{F W} \mathbf{F}^{-1} \mathbf{s} \\
& +\frac{1}{2} J_{0} h_{1}^{2} m_{3}^{(f)}(1-\bar{v}) \frac{\partial}{\partial \xi^{\alpha}}\left(\mathbf{F W F} \mathbf{F}^{-1}\right) \mathbf{F}(\overline{\mathbf{u}} \otimes \overline{\mathbf{u}}) \mathbf{e}_{\alpha} \\
& +J_{0} h_{1}^{2} \beta_{f}(1-\overline{\mathrm{v}}) \mathbf{F} \mathbf{W} \overline{\mathbf{u}}-\frac{c_{s f} J_{0} h_{1}^{2}}{2\left(c_{s f} \overline{\mathrm{v}}+1-\overline{\mathrm{v}}\right)} \\
& \times\left\{c+a_{s} m_{3}^{(s)}(1-\bar{v})+a_{f} m_{3}^{(f)} \bar{v}\right\} \mathbf{F} \mathbf{M}_{0} \operatorname{Grad} \bar{v} \\
& -J_{0} h_{1} h\left(c+a_{2} m_{2}^{(2)}\right) \mathbf{F} \mathbf{M}_{0} \operatorname{Grad} \bar{v} \\
& -c_{s f} c_{D} J_{0} h_{1}\left\{\left(1-\Omega h_{1} m_{1}^{(f)}\right) \mathbf{F} \overline{\mathbf{u}}-\left(1-\Omega h_{1} m_{1}^{(s)}\right) \mathbf{F} \overline{\mathbf{v}}\right\},
\end{aligned}
$$

with $\quad \tilde{a}_{f} \equiv \mathbf{H} \overline{\mathbf{u}} \cdot \mathbf{W} \overline{\mathbf{u}}, \quad \bar{p}_{f}$ as given by $(81)_{2}$, and $s_{\text {int }}$ deduced from (65) as

$$
\begin{aligned}
& s_{\text {int }}=-\frac{\partial}{\partial t}\left\{J_{0} h_{1}\left(1-\Omega h_{1}\right)(1-\overline{\mathrm{v}})\right\} \\
& -\operatorname{Div}\left\{J_{0} h_{1}\left(1-n_{1}^{(f)} \overline{\mathrm{v}}-m_{1}^{(f)}(1-\overline{\mathrm{v}}) \Omega h_{1}\right) \overline{\mathbf{u}}\right\}
\end{aligned}
$$

- The depth-integrated mass and tangential momentum balances (67), (70) for the fluid in $\mathcal{E}_{2}$ emerge as

$$
\begin{aligned}
\mathcal{M}_{2}= & s_{\text {int }}, \\
\mathcal{L}_{2}= & \mathcal{R}_{2}+\mathbf{F}\left\{J\left[\left(-p_{2} \mathbf{M}+\mathbf{P}_{2}\right) \operatorname{Grad} h_{1}-\mathbf{p}_{2}\right]\right\}_{\xi=h_{1}} \\
& +\left.s_{\text {int }} \mathbf{F} \mathbf{v}_{2}\right|_{\xi=h_{1}},
\end{aligned}
$$

where 


$$
\begin{aligned}
\mathcal{M}_{2} \equiv & \frac{\partial}{\partial t}\left\{J_{0} h\left[1-\Omega\left(h_{1}+h_{2}\right)\right]\right\} \\
& +\operatorname{Div}\left\{J_{0} h\left[1-\Omega\left(h_{1}+h_{2}\right) m_{1}^{(2)}\right] \overline{\mathbf{v}}_{2}\right\}, \\
\mathcal{L}_{2} \equiv & \frac{\partial}{\partial t}\left\{J_{0} h\left[1-\Omega\left(h_{1}+h_{2}\right) m_{1}^{(2)}\right] \mathbf{F} \mathbf{v}_{2}\right\} \\
& +\operatorname{Div}\left\{J _ { 0 } h \mathbf { F } \left[\left(m_{2}^{(2)}-\Omega\left(h_{1}+h_{2}\right) m_{3}^{(2)}\right) \overline{\mathbf{v}}_{2} \otimes \overline{\mathbf{v}}_{2}\right.\right. \\
& \left.\left.+\bar{p}_{2} \mathbf{M}_{0}-\overline{\mathbf{P}}_{2}\right]\right\}+J_{0} h\left\{2 \mathbf{F W} \overline{\mathbf{p}}_{2}-\left(\mathbf{H} \cdot \overline{\mathbf{P}}_{2}\right) \mathbf{s}\right\}, \\
\mathcal{R}_{2} \equiv & -J_{0} h\left\{2 \Omega \bar{p}_{2}+c\left(1-\Omega\left(h_{1}+h_{2}\right)\right)+a_{2} m_{2}^{(2)}\right. \\
& \left.-\Omega\left(h_{1}+h_{2}\right) a_{2} m_{3}^{(2)}-\frac{1}{2}\left(h_{1}+h_{2}\right) \tilde{a}_{2} m_{3}^{(2)}\right\} \mathbf{s} \\
& -\frac{1}{2} J_{0} h\left(h_{1}+h_{2}\right)\left(c+a_{2} m_{3}^{(2)}\right) \mathbf{F W} \mathbf{F}^{-1} \mathbf{s} \\
& +\frac{1}{2} J_{0} h\left(h_{1}+h_{2}\right) m_{3}^{(2)} \frac{\partial}{\partial \xi^{\alpha}}\left(\mathbf{F W F} \mathbf{F}^{-1}\right) \mathbf{F}\left(\overline{\mathbf{v}}_{2} \otimes \overline{\mathbf{v}}_{2}\right) \mathbf{e}_{\alpha} \\
& +2 J_{0} h\left\{\frac{\partial h_{2}}{\partial t}+\operatorname{Grad} h_{2} \cdot \overline{\mathbf{v}}_{2}\right\} \mathbf{F W} \overline{\mathbf{v}}_{2}+J_{0} h^{2} \beta_{2} \mathbf{F W} \overline{\mathbf{v}}_{2},
\end{aligned}
$$

with $\quad \tilde{a}_{2} \equiv \mathbf{H} \overline{\mathbf{v}}_{2} \cdot \mathbf{W} \overline{\mathbf{v}}_{2}$, and $\bar{p}_{2}$ as given by $(80)_{1}$.

The 8 scalar shallow flow Eqs. (83), (84), (85), (87), (88) stand, under suitable closure relations, for the determination of the 9 unknown scalar fields $\bar{v}, \overline{\mathbf{v}}, \overline{\mathbf{u}}$, $\overline{\mathbf{v}}_{\mathbf{2}}, h_{1}, h_{2}$. Clearly, an additional equation is needed. For immiscible layers this equation is $s_{i n t}=0$, see (57). This suggests, for the general case, that apart from the classical closure relations a parameterization for $s_{\text {int }}$ must be prescribed. When combined with (86) this gives the necessary equation, see Sect. 5 .

Next we consider 2 cases for which the shallow flow equations are further simplified.

\section{Case 1) Small Curvature}

If the basal topography exhibits small curvature, in the sense that $\mathcal{H}=O\left(\epsilon^{\gamma}\right)$, many terms in the shallow flow equations are $O\left(\epsilon^{2+\gamma}\right)$, and hence negligible. Moreover, $a_{2}=O\left(\epsilon^{1+\gamma}\right)$ yields $\bar{p}_{2}=c h / 2$, and this together with

$\operatorname{Div} \mathbf{F} \mathbf{M}_{0}=-\operatorname{tr}\left(\mathbf{F}^{-1} \frac{\partial \mathbf{F}}{\partial \xi^{\alpha}}\right) \mathbf{F} \mathbf{M}_{0} \mathbf{e}_{\alpha}-\mathbf{F W F}^{-1} \mathbf{s}-2 \Omega \mathbf{s}$,

Grad $J_{0}=J_{0} \operatorname{tr}\left(\mathbf{F}^{-1} \frac{\partial \mathbf{F}}{\partial \xi^{\alpha}}\right) \mathbf{e}_{\alpha}+J_{0} \mathbf{H F}^{-1} \mathbf{s}$,

$\operatorname{Grad} c=-c \mathbf{H F}^{-1} \mathbf{s}$,

see [27], can be used to re-write $\operatorname{Div}\left(J_{0} h_{1} \bar{p}_{s} \mathbf{F} \mathbf{M}_{0}\right)$ and similar terms in $\mathcal{L}_{s, f, 2}$. Thus, up to $O\left(\epsilon^{2+\gamma}\right)$, the shallow flow equations read as follows:

- Equations (83), (84) for the solid constituent in the lower layer turn, respectively, into

$$
\frac{\partial}{\partial t}\left\{J_{0} h_{1} \bar{v}\right\}+\operatorname{Div}\left\{J_{0} h_{1} n_{1}^{(s)} \bar{v} \overline{\mathbf{v}}\right\}=0
$$

$\frac{\partial}{\partial t}\left\{J_{0} h_{1} n_{1}^{(s)} \bar{v} \mathbf{F} \overline{\mathbf{v}}\right\}+\operatorname{Div}\left\{J_{0} h_{1} \mathbf{F}\left[n_{2}^{(s)} \overline{\mathbf{v}} \overline{\mathbf{v}} \otimes \overline{\mathbf{v}}-\overline{\mathbf{P}}_{s}\right]\right\}$

$+J_{0} \mathbf{F} \mathbf{M}_{0} \operatorname{Grad}\left(h_{1} \bar{p}_{s}\right)+2 J_{0} h_{1} \mathbf{F} \mathbf{W} \overline{\mathbf{p}}_{s}=-\left.J_{0} \mathbf{F} \mathbf{p}_{s}\right|_{\xi=0}$

$-\frac{1}{c_{s f}} \mathbf{F}\left\{J \cup\left[\left(-p_{2} \mathbf{M}+\mathbf{P}_{2}\right) \text { Grad } h_{1}-\mathbf{p}_{2}\right]\right\}_{\xi=h_{1}}$

$-J_{0} h_{1}\left(c+a_{s} n_{2}^{(s)}\right) \bar{v} \mathbf{s}+c_{D} J_{0} h_{1} \mathbf{F}(\overline{\mathbf{u}}-\overline{\mathbf{v}})$

$+J_{0} h_{1} c\left\{\frac{h_{1}}{2\left(c_{s f} \overline{\mathrm{v}}+1-\overline{\mathrm{v}}\right)}+\frac{h}{c_{s f}}\right\} \mathbf{F} \mathbf{M}_{0} \operatorname{Grad} \overline{\mathrm{v}}$,

where, owing to $(81)_{1}$ and $a_{s}, a_{2}=O\left(\epsilon^{\gamma}\right)$,

$$
\bar{p}_{s}=\bar{v} c\left(\frac{1}{2} h_{1}+\frac{1}{c_{s f}} h\right) .
$$

- Equation (85) for the fluid constituent in the lower layer emerges as

$$
\begin{aligned}
& \frac{\partial}{\partial t}\left\{J_{0} h_{1}\left(1-n_{1}^{(f)} \overline{\mathrm{v}}\right) \mathbf{F} \overline{\mathbf{u}}\right\} \\
& +\operatorname{Div}\left\{J_{0} h_{1} \mathbf{F}\left[\left(m_{2}^{(f)}-n_{2}^{(f)} \overline{\mathrm{v}}\right) \overline{\mathbf{u}} \otimes \overline{\mathbf{u}}-\overline{\mathbf{P}}_{f}\right]\right\} \\
& +J_{0} \mathbf{F} \mathbf{M}_{0} \operatorname{Grad}\left(h_{1} \bar{p}_{f}\right)+2 J_{0} h_{1} \mathbf{F} \mathbf{W} \overline{\mathbf{p}}_{f}=-\left.J_{0} \mathbf{F} \mathbf{p}_{f}\right|_{\xi=0} \\
& -\mathbf{F}\left\{J(1-v)\left[\left(-p_{2} \mathbf{M}+\mathbf{P}_{2}\right) \operatorname{Grad} h_{1}-\mathbf{p}_{2}\right]\right\}_{\xi=h_{1}} \\
& -J_{0} h_{1}\left\{c(1-\overline{\mathrm{v}})+a_{f}\left(m_{2}^{(f)}-n_{2}^{(f)} \overline{\mathrm{v}}\right)\right\} \mathbf{s} \\
& -J_{0} h_{1} c\left\{\frac{c_{s f} h_{1}}{2\left(c_{s f} \overline{\mathrm{v}}+1-\overline{\mathrm{v}}\right)}+h\right\} \mathbf{F} \mathbf{M}_{0} \operatorname{Grad} \overline{\mathrm{v}} \\
& -c_{s f} c_{D} J_{0} h_{1} \mathbf{F}(\overline{\mathbf{u}}-\overline{\mathbf{v}})-\left.s_{i n t} \mathbf{F} \mathbf{v}_{2}\right|_{\xi=h_{1}},
\end{aligned}
$$

where, see (81) $)_{2}$ and (86) with $a_{f}, a_{2}, \Omega=O\left(\epsilon^{\gamma}\right)$,

$$
\begin{aligned}
\bar{p}_{f} & =(1-\bar{v}) c\left(\frac{1}{2} h_{1}+h\right), \\
s_{\text {int }} & =-\frac{\partial}{\partial t}\left\{J_{0} h_{1}(1-\bar{v})\right\}-\operatorname{Div}\left\{J_{0} h_{1}\left(1-n_{1}^{(f)} \bar{v}\right) \overline{\mathbf{u}}\right\} .
\end{aligned}
$$

- Equations (87), (88) for the upper layer read

$\frac{\partial}{\partial t}\left\{J_{0} h\right\}+\operatorname{Div}\left\{J_{0} h \overline{\mathbf{v}}_{2}\right\}=s_{\text {int }}$,

$$
\begin{aligned}
& \frac{\partial}{\partial t}\left\{J_{0} h \mathbf{F} \overline{\mathbf{v}}_{2}\right\}+\operatorname{Div}\left\{J_{0} h \mathbf{F}\left[m_{2}^{(2)} \overline{\mathbf{v}}_{2} \otimes \overline{\mathbf{v}}_{2}-\overline{\mathbf{P}}_{2}\right]\right\} \\
& \quad+J_{0} h \mathbf{F} \mathbf{M}_{0} \operatorname{Grad} c h+2 J_{0} h \mathbf{F} \mathbf{W} \overline{\mathbf{p}}_{2} \\
& =\mathbf{F}\left\{J\left[\left(-p_{2} \mathbf{M}+\mathbf{P}_{2}\right) \operatorname{Grad} h_{1}-\mathbf{p}_{2}\right]\right\}_{\xi=h_{1}} \\
& \quad-J_{0} h\left(c+a_{2} m_{2}^{(2)}\right) \mathbf{s}+\left.s_{i n t} \mathbf{F} \mathbf{v}_{2}\right|_{\xi=h_{1}} .
\end{aligned}
$$

In the above equations one can see that there are only a few terms which explicitly account for the curvature of the basal surface, namely, those which contain the matrix $\mathbf{W}$ and the scalars $a_{s}, a_{f}, a_{2}$. However, the curvature may also occur in the closure relations, see e.g. the Coulomb basal friction law (100) below.

\section{Case 2) Negligible Slip Between Solid and Fluid at Small Basal Curvature}

As argued by Iverson and Denlinger [31], see also Takahashi [2], a simplifying assumption in a fully de- 
veloped flow of a solid-fluid mixture is $\|\boldsymbol{u}-\boldsymbol{v}\|<<$ $\|\boldsymbol{v}\|$ : the granular solid moves nearly with the velocity of the adjacent fluid. Hence, the momentum balance equation of the mixture as a whole should be used instead of the two momentum balances for the constituents. We assume

$$
\mathbf{u}=\mathbf{v}+O\left(\epsilon^{1+\gamma}\right)
$$

which implies satisfaction of the condition $\|\boldsymbol{u}-\boldsymbol{v}\|<<$ $\|\boldsymbol{v}\|$ in the sense $O\left(\epsilon^{2}\right)<<\mathbf{M}^{-1} \mathbf{v} \cdot \mathbf{v}^{3}$, and use of the depth-averaged tangential momentum balance of the mixture as a whole instead of the depth-averaged tangential momentum balance of each mixture constituent. Note, (97) yields, up to $O\left(\epsilon^{1+\gamma}\right)$ terms, the same Boussinesq coefficients for the pore fluid and the solid, i.e.,

$$
\begin{aligned}
& n_{1}^{(f)}=n_{1}^{(s)}, n_{2}^{(f)}=n_{2}^{(s)}, \\
& m_{1}^{(f)}=m_{1}^{(s)}, m_{2}^{(f)}=m_{2}^{(s)}, m_{3}^{(f)}=m_{3}^{(s)} .
\end{aligned}
$$

For simplicity we refer to the case of small curvature $\mathcal{H}$ $=O\left(\epsilon^{\gamma}\right)$ of the basal surface, and hence the depthaveraged tangential momentum balance of the mixture as a whole is $c_{s f} \times(91)+(93)$, which emerges as

$$
\begin{aligned}
& \frac{\partial}{\partial t}\left\{J_{0} h_{1}\left(\left(c_{s f}-1\right) n_{1}^{(s)} \overline{\mathrm{v}}+1\right) \mathbf{F} \overline{\mathbf{v}}\right\} \\
& +\operatorname{Div}\left\{J_{0} h_{1} \mathbf{F}\left[\left(\left(c_{s f}-1\right) n_{1}^{(s)} \overline{\mathrm{v}}+m_{2}^{(s)}\right) \overline{\mathbf{v}} \otimes \overline{\mathbf{v}}-c_{s f} \overline{\mathbf{P}}_{s}-\overline{\mathbf{P}}_{f}\right]\right\} \\
& +J_{0} \mathbf{F} \mathbf{M}_{0} \operatorname{Grad}\left\{h_{1}\left(c_{s f} \bar{p}_{s}+\bar{p}_{f}\right)\right\} \\
& +2 J_{0} h_{1} \mathbf{F W}\left(c_{s f} \overline{\mathbf{p}}_{s}+\overline{\mathbf{p}}_{f}\right)=-J_{0} \mathbf{F}\left(c_{s f} \mathbf{p}_{s}+\mathbf{p}_{f}\right)_{\xi=0} \\
& -\mathbf{F}\left\{J\left[\left(-p_{2} \mathbf{M}+\mathbf{P}_{2}\right) \operatorname{Grad} h_{1}-\mathbf{p}_{2}\right]\right\}_{\xi=h_{1}} \\
& -J_{0} h_{1}\left\{c\left(c_{s f} \overline{\mathrm{v}}+1-\overline{\mathrm{v}}\right)\right. \\
& \left.+a_{s}\left(\left(c_{s f}-1\right) n_{2}^{(s)} \overline{\mathrm{v}}+m_{2}^{(s)}\right)\right\} \mathbf{s}-\left.s_{i n t} \mathbf{F} \mathbf{v}_{2}\right|_{\xi=h_{1}},
\end{aligned}
$$

where $a_{f}=a_{s}+O\left(\epsilon^{1+\gamma}\right)$ has been used (see definitions after (81) and (97)), and $\bar{p}_{s}, \bar{p}_{f}$ are given by (92), $(94)_{1}$, respectively. Moreover, in view of (90), the fluid mass flux $s_{\text {int }}$ in $(94)_{2}$ is given by

$$
s_{\text {int }}=-\frac{\partial}{\partial t}\left\{J_{0} h_{1}\right\}-\operatorname{Div}\left\{J_{0} h_{1} \overline{\mathbf{v}}\right\} .
$$

Therefore, under assumption (97), the equations describing the motion of the two-layer system on a surface with $\mathcal{H}=O\left(\epsilon^{\gamma}\right)$ are as follows:

- the depth-averaged mass balance Eq. (90) for the solid constituent;

- the depth-averaged tangential momentum balance Eq. (98) for the mixture as a whole;

- the depth-averaged mass and tangential momentum balances (95), (96) for the upper layer.

The mean pressures $\bar{p}_{s, f}$ are given by (92), (94) 1 , and

$$
\begin{aligned}
& \text { Indeed, }\|\boldsymbol{u}-\boldsymbol{v}\|^{2}=\mathbf{M}^{-1}(\mathbf{u}-\mathbf{v}) \cdot(\mathbf{u}-\mathbf{v})+(\mathrm{u}-\mathrm{v})^{2}, \\
& \|\boldsymbol{v}\|^{2}=\mathbf{M}^{-1} \mathbf{v} \cdot \mathbf{v}+\mathrm{v}^{2}, \mathbf{v}=O(1), \mathbf{u}=O(1), \mathbf{v}=O(\epsilon), \\
& \mathbf{u}=O(\epsilon), \text { and one uses the assumption } \mathbf{u}=\mathbf{v}+O\left(\epsilon^{1+\gamma}\right)
\end{aligned}
$$

$s_{\text {int }}$ can be found in (99).

The above mentioned equations stand for the determination of the 7 basic unknowns $\bar{v}, \overline{\mathbf{v}}, \overline{\mathbf{v}}_{\mathbf{2}}, h_{1}, h_{2}$. For immiscible layers equation $s_{\text {int }}=0$ must also be taken into account, and for miscible layers an additional closure condition is needed, see Sect. 4.2.

If there is no mass exchange at the layer interface, one may assume $v=$ constant. If so, since now $n_{1}^{(s)}=1$, equations $s_{\text {int }}=0$ and (90) coincide, and the depth-averaged tangential momentum balance equation of the mixture as a whole, (98), is recast into

$$
\begin{aligned}
& \frac{\partial}{\partial t}\left\{J_{0} h_{1}\left(c_{s f} \overline{\mathrm{v}}+1-\overline{\mathrm{v}}\right) \mathbf{F} \overline{\mathbf{v}}\right\} \\
& +\operatorname{Div}\left\{J_{0} h_{1} \mathbf{F}\left[\left(c_{s f} \overline{\mathrm{v}}+1-\overline{\mathrm{v}}\right) m_{2}^{(s)} \overline{\mathbf{v}} \otimes \overline{\mathbf{v}}-c_{s f} \overline{\mathbf{P}}_{s}-\overline{\mathbf{P}}_{f}\right]\right\} \\
& +J_{0} \mathbf{F} \mathbf{M}_{0} \operatorname{Grad}\left\{h_{1}\left(c_{s f} \bar{p}_{s}+\bar{p}_{f}\right)\right\} \\
& +2 J_{0} h_{1} \mathbf{F W}\left(c_{s f} \overline{\mathbf{p}}_{s}+\overline{\mathbf{p}}_{f}\right)=-J_{0} \mathbf{F}\left(c_{s f} \mathbf{p}_{s}+\mathbf{p}_{f}\right)_{\xi=0} \\
& -\mathbf{F}\left\{J\left[\left(-p_{2} \mathbf{M}+\mathbf{P}_{2}\right) \operatorname{Grad} h_{1}-\mathbf{p}_{2}\right]\right\}_{\xi=h_{1}} \\
& -J_{0} h_{1}\left(c_{s f} \overline{\mathrm{v}}+1-\overline{\mathrm{v}}\right)\left(c+a_{s} m_{2}^{(s)}\right) \mathbf{s},
\end{aligned}
$$

where $n_{2}^{(s)}=m_{2}^{(s)}$, which holds since $v=$ constant has been used. A similar approach can be found in the paper by Fernández-Nieto et al. [6] for the description of a one-dimensional shallow flow of two immiscible layers.

\section{CLOSURE RELATIONS}

\subsection{The Stress Terms}

The shallow flow Eqs. (83), (84), (85), (87), (88) or their simplified forms corresponding to cases 1), 2) above, must be complemented by closure relations for

$$
\overline{\mathbf{P}}_{s}, \overline{\mathbf{P}}_{f}, \overline{\mathbf{P}}_{2}, \overline{\mathbf{p}}_{s}, \overline{\mathbf{p}}_{f}, \overline{\mathbf{p}}_{2},\left.\mathbf{p}_{s}\right|_{\xi=0},\left.\mathbf{p}_{f}\right|_{\xi=0},
$$

as well as for

$$
\left\{\left(-p_{2} \mathbf{M}+\mathbf{P}_{2}\right) \operatorname{Grad} h_{1}-\mathbf{p}_{2}\right\}_{\xi=h_{1}},\left.\quad v\right|_{\xi=h_{1}} .
$$

We must also specify the pressures $p_{s}, p_{f}, p_{2}$. Besides, when there is mass exchange at the layer interface, a scalar equation, e.g., analogous to $s_{\text {int }}=0$, is needed.

For the solid constituent we assume the closure relations proposed [27]. Accordingly, $p_{s} \equiv-\sigma_{s} \boldsymbol{n} \cdot \boldsymbol{n}$, the bulk granular material is modelled as (1) an inviscid fluid, or as (2) a topography-adapted version of the Iverson-Denlinger model, or as (3) a topographyadapted version of the Savage-Hutter model (see [27] for details), and the shear stresses $\mathbf{p}_{s} \mid \xi=0$ are derived from the assumption of the classical basal Coulomb friction as

$$
\left.\mathbf{p}_{s}\right|_{\xi=0}=(\tan \delta)\left(\left.p_{s}\right|_{\xi=0}\right)+\operatorname{sgn}\left(\left.\mathbf{v}\right|_{\xi=0}\right) .
$$

In (100) the index + stands for the positive part of a quantity, i.e., $f_{+} \equiv \max \{0, f\}$; for a 2 -column $\mathbf{x}$ the multivalued function sgn $\mathbf{x}$ is defined as 


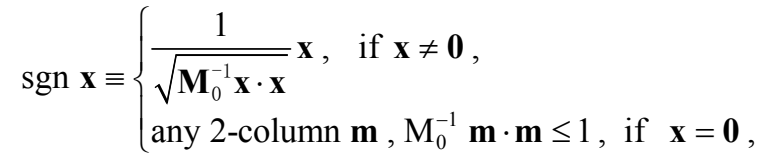

the pressure $p_{s \mid \xi=0}$ is given by (82), and $\delta>0$ is the basal angle of friction. We assume

$$
\tan \delta=O\left(\epsilon^{\gamma}\right),\left.\quad \mathbf{v}\right|_{\xi=0}=\chi_{s} \overline{\mathbf{v}}, \quad \chi_{s} \in(0,1] .
$$

The fluid in both layers is supposed to be Newtonian with small viscosity, $\eta=O\left(\epsilon^{2+\gamma}\right)$ (water is such a fluid). As a consequence, see e.g. [20],

$$
\mathbf{P}_{f, 2}=O\left(\epsilon^{2+\gamma}\right), \quad \mathbf{p}_{f, 2}=O\left(\epsilon^{1+\gamma}\right), \quad P_{f, 2}^{33}=O\left(\epsilon^{2+\gamma}\right),
$$

and hence the ordering approximations (76) are satisfied. Moreover, the terms containing $\overline{\mathbf{P}}_{f}, \overline{\mathbf{p}}_{f}, \overline{\mathbf{P}}_{2}$, $\overline{\mathbf{p}}_{2}$ in the shallow flow equations referring to the fluid in both layers are negligibly small. Then, the basal shear stresses $\left.\mathbf{p}_{f}\right|_{\xi=0}$ are obtained as in [27] by postulating the viscous basal friction law (dimensionless form):

$$
\boldsymbol{\sigma}_{f} \boldsymbol{n}-\left(\boldsymbol{\sigma}_{f} \boldsymbol{n} \cdot \boldsymbol{n}\right) \boldsymbol{n}=(1-v) c_{\text {base }}\|\boldsymbol{u}\| \boldsymbol{u} \text { at } \xi=0,
$$

with constant friction coefficient $c_{\text {base }}$. Thus, with the assumption

$$
\left.\mathbf{u}\right|_{\xi=0}=\chi_{f} \overline{\mathbf{u}}, \quad \chi_{f} \in(0,1]
$$

and neglecting $O\left(\epsilon^{2+\gamma}\right)$ terms, this yields

$$
\left.\mathbf{p}_{f}\right|_{\xi=0}=c_{\text {base }} \chi_{f}^{2}(1-\overline{\mathrm{v}}) \sqrt{\mathbf{M}_{0}^{-1} \overline{\mathbf{u}} \cdot \overline{\mathbf{u}}} \overline{\mathbf{u}} .
$$

The friction coefficient $c_{\text {base }}$ and the coefficient $\mathcal{X}_{f}$ are such that $c_{\text {base }} \mathcal{X}_{f}^{2}=O\left(\epsilon^{1+\gamma}\right)$, since $\mathbf{p}_{f}$ on the left-hand side of (102) is of this order.

At the layer interface $\xi=h_{1}$, the shear stress of the fluid is assumed to follow a Chézy-like viscous friction law, which in non-dimensional form reads

$$
\begin{aligned}
& \boldsymbol{\sigma}_{2} \boldsymbol{n}_{1}-\left(\boldsymbol{\sigma}_{2} \boldsymbol{n}_{1} \cdot \boldsymbol{n}_{1}\right) \boldsymbol{n}_{1} \\
& =c_{i n t}\left\|\boldsymbol{v}_{2 \tau}-\tilde{\boldsymbol{v}}_{1 \tau}\right\|\left(\boldsymbol{v}_{2 \tau}-\tilde{\boldsymbol{v}}_{1 \tau}\right), \\
& \boldsymbol{v}_{2 \tau} \equiv \boldsymbol{v}_{2}-\left(\boldsymbol{v}_{2} \cdot \boldsymbol{n}_{1}\right) \boldsymbol{n}_{1}, \tilde{\boldsymbol{v}}_{1 \tau} \equiv \tilde{\boldsymbol{v}}_{1}-\left(\tilde{\boldsymbol{v}}_{1} \cdot \boldsymbol{n}_{1}\right) \boldsymbol{n}_{1}, \\
& \tilde{\boldsymbol{v}}_{1} \equiv v \boldsymbol{v}+(1-\mathrm{v}) \boldsymbol{u} ;
\end{aligned}
$$

$c_{i n t}>0$ is assumed constant and of the same order as the left-hand side of (103), i.e., $O\left(\epsilon^{1+\gamma}\right)$, see (101). Since $\left\|\nabla F_{1}\right\|^{2}=1+\mathbf{M} \operatorname{Grad} h_{1} \cdot \operatorname{Grad} h_{1}$, see (53), we have $\left\|\nabla F_{1}\right\|=1+O\left(\epsilon^{2}\right)$, which, in view of approximations (101), transforms the left-hand side of (103) into

$$
\left(\mathbf{p}_{2}, 0\right)^{T}+O\left(\epsilon^{2+\gamma}\right)
$$

Then, $\mathcal{U}_{1}=\left.\boldsymbol{v}_{1}\right|_{\xi=h_{1}} \cdot \boldsymbol{n}_{1}$ and definition (29) yield (36) as $\boldsymbol{v}_{2} \cdot \boldsymbol{n}_{1}=\tilde{\boldsymbol{v}}_{1} \cdot \boldsymbol{n}_{1}$ at $\boldsymbol{\xi}=h_{1}$, and hence

$$
\boldsymbol{v}_{2 \tau}-\tilde{\boldsymbol{v}}_{1 \tau}=\boldsymbol{v}_{2}-\tilde{\boldsymbol{v}}_{1}=\left(\mathrm{v}_{2}^{\alpha}-\tilde{\mathrm{v}}_{1}^{\alpha}\right) \boldsymbol{g}_{\alpha}+O(\epsilon),
$$

so that, with $c_{i n t}=O\left(\epsilon^{1+\gamma}\right)$ and the assumption

$$
\left.\left(\mathbf{v}_{2}-\tilde{\mathbf{v}}_{1}\right)\right|_{\xi=h_{1}}=\chi_{\text {int }}\left(\overline{\mathbf{v}}_{2}-\overline{\tilde{\mathbf{v}}}_{1}\right), \quad \chi_{\text {int }}>0,
$$

(103) turns into

$$
\begin{aligned}
\left.\mathbf{p}_{2}\right|_{\xi=h_{1}}= & c_{\text {int }} \chi_{\text {int }}^{2} \sqrt{\mathbf{M}_{0}^{-1}\left(\overline{\mathbf{v}}_{2}-\overline{\tilde{\mathbf{v}}}_{1}\right) \cdot\left(\overline{\mathbf{v}}_{2}-\overline{\tilde{\mathbf{v}}}_{1}\right)}\left(\overline{\mathbf{v}}_{2}-\overline{\tilde{\mathbf{v}}}_{1}\right) \\
& +O\left(\epsilon^{2+\gamma}\right) .
\end{aligned}
$$

Consequently, apart from $O\left(\epsilon^{2+\gamma}\right)$ terms, we have

$$
\begin{aligned}
\{J[ & \left.\left.\left(-p_{2} \mathbf{M}+\mathbf{P}_{2}\right) \operatorname{Grad} h_{1}-\mathbf{p}_{2}\right]\right\}_{\xi=h_{1}} \\
= & -J_{0} h\left(c+a_{2} m_{2}^{(2)}\right) \mathbf{M}_{0} \operatorname{Grad} h_{1} \\
& \quad-J_{0} c_{\text {int }} \chi_{\text {int }}^{2} \sqrt{\mathbf{M}_{0}^{-1}\left(\overline{\mathbf{v}}_{2}-\overline{\tilde{\mathbf{v}}}_{1}\right) \cdot\left(\overline{\mathbf{v}}_{2}-\overline{\tilde{\mathbf{v}}}_{1}\right)}\left(\overline{\mathbf{v}}_{2}-\overline{\tilde{\mathbf{v}}}_{1}\right)
\end{aligned}
$$

Recalling that $\mathrm{v}=\overline{\mathrm{v}}+O(\epsilon)$ and $c_{i n t}=O\left(\epsilon^{1+\gamma}\right)$, we see that in the preceding Chézy relation the mean velocity $\overline{\tilde{\mathrm{V}}}_{1}$ can be replaced by

$$
\overline{\tilde{\mathbf{v}}}_{1}=\overline{\mathrm{v}} \overline{\mathbf{v}}+(1-\overline{\mathrm{v}}) \overline{\mathbf{u}}+O(\epsilon) .
$$

For small curvature $\mathcal{H}=O\left(\epsilon^{\gamma}\right)$, we have $\operatorname{Grad} c=O\left(\epsilon^{\gamma}\right)$, and so (104) can be written as

$$
\begin{aligned}
& \left\{J\left[\left(-p_{2} \mathbf{M}+\mathbf{P}_{2}\right) \operatorname{Grad} h_{1}-\mathbf{p}_{2}\right]\right\}_{\xi=h_{1}} \\
& =-J_{0} h \mathbf{M}_{0} \operatorname{Grad} c h_{1} \\
& -J_{0} c_{\text {int }} \chi_{\text {int }}^{2} \sqrt{\mathbf{M}_{0}^{-1}\left(\overline{\mathbf{v}}_{2}-\overline{\tilde{\mathbf{v}}}_{1}\right) \cdot\left(\overline{\mathbf{v}}_{2}-\overline{\tilde{\mathbf{v}}}_{1}\right)}\left(\overline{\mathbf{v}}_{2}-\overline{\tilde{\mathbf{v}}}_{1}\right) .
\end{aligned}
$$

Now we refer to $\left.v\right|_{\xi=h_{1}}$. In view of (74),

$$
\left.v\right|_{\xi=h_{1}}=\bar{v}+O(\epsilon),
$$

and with $\sigma_{2}^{E}=O\left(\epsilon^{1+\gamma}\right)$, it is clear that $\left.v\right|_{\xi=h_{1}}$ can be replaced by $\bar{v}$ in (84) and (85). However, for some flows (106) may be a too rough approximation for the volume fraction at the layer interface. Indeed, data of Egashira et al. [32] show that in a water-sediment mixture the solid volume fraction $v$ decreases abruptly to a value close to zero near the free surface. Similar behavior for $v$ in the two-layer flow is likely, see Fig. 3, so, we may take relation (74) as an acceptable approximation whenever $v$ occurs under the integral from 0 to $h_{1}$ and when $\left.v\right|_{\xi=0}$ has to be evaluated, and assign a value as determined from experiments to $v \mid \xi=h_{1}$. Note, the only terms in which $v$ is not under the integral sign are $\left.p_{s}\right|_{\xi=0}$ and those evaluated at $\xi=h_{1}$ in (84), (85).

The formulation of the closure relations is now complete for the case that there is no fluid mass exchange at the layer interface, because $s_{\text {int }}=0$ yields the necessary number of equations for $\overline{\mathrm{v}}, \overline{\mathbf{v}}, \overline{\mathbf{u}}, \overline{\mathbf{v}}_{\mathbf{2}}, h_{1}, h_{2}$.

\subsection{Mass Flux Across the Layer Interface- A Challenge}

For the case that fluid mass flux across the layer interface occurs, there are 8 scalar Eqs. $((83),(84),(85)$, 


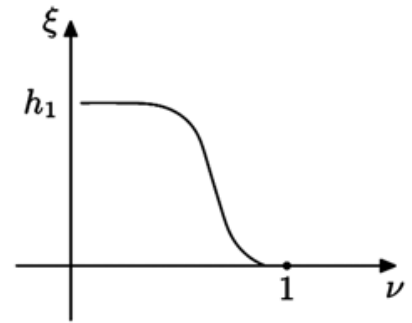

Fig. 3 Conjectured experimental curve for the distribution of the solid volume fraction

(87), (88)), complemented by closure relations as given in Sect. 4.1, for the 9 basic unknown scalar fields $\bar{v}$, $\overline{\mathbf{v}}, \overline{\mathbf{u}}, \overline{\mathbf{v}}_{\mathbf{2}}, h_{1}, h_{2}$. Therefore, one more closure equation is needed. Next we make a few suggestions for it.

1. A so-called saturation pressure $\varpi$ has been used by $\mathrm{Wu}$ et al. [13] in their two-layer model for an ice-sediment mixture, to give closure relations for $p_{s}$, $p_{f}$ in terms of $\varpi$ and $v$. Applying this idea to our two-layer system, we could introduce the saturation pressure $\varpi$, and two equations, obtained by combining the closure relations for $p_{s}, p_{f}$ with (81). The question is then how to choose the dependence of $p_{s}$, $p_{f}$ on $\varpi, v$.

For instance, we could use the pressure equilibrium assumption, which, in dimensional form, requests

$$
p_{s}=v \varpi, p_{f}=(1-v) \varpi \Leftrightarrow \tilde{p}_{s}=\tilde{p}_{f} .
$$

However, for small curvature $\mathcal{H}=O\left(\epsilon^{\gamma}\right)$ of the basal topography, one can easily show that (107) and (92), $(94)_{1}$ give $\tilde{\rho}_{s}=\tilde{\rho}_{f}$, while in debris flows typically we have $\tilde{\rho}_{s} \approx 2.5 \tilde{\rho}_{f}$. That is, pressure equilibrium can not play the role of the additional equation that we need. In passing we mention that Schneider and Hutter [33] pointed out that (107) has no theoretical basis other than rendering a closed system of equations. Most likely, a thermodynamic analysis can deliver the dependence of $p_{s}, p_{f}$ on $\varpi, v$, see e.g., Wu et al. [13], Schneider and Hutter [33].

2. Another possibility of choosing the additional equation consists in combining formula $(81)_{1}$ for the mean solid pressure $\bar{p}_{s}$ with the prescription of a constitutive relation for $p_{s}$ (see e.g. Egashira et al. [32], Takahama et al. [7], Takahashi [2] for the closure condition on $p_{s}$ ).

3. Assuming the distribution of the solid volume fraction $v$, see e.g., Lien and Tsai [34], is another way to close the system of modeling equations for the twolayer flow.

4. A closure relation for the mass flux $\mathcal{M}_{\text {int }}$ at the layer interface is also an option. This way we can see the condition $\mathcal{M}_{\text {int }}=0$ for immiscible layers as a particular closure relation for $\mathcal{M}_{\text {int }}$. In view of (56), the closure relation for $\mathcal{M}_{\text {int }}$ gives a closure relation for $s_{\text {int }}$. According to (21), an estimation of $\mathcal{M}_{\text {int }}$ reduces to that of the solid volume fraction and of the relative velocity normal to the surface $\mathcal{S}_{1}$ of the two mixture constituents, evaluated on the layer interface. Giving a law for $\mathcal{M}_{\text {int }}$ is similar to giving a law for the erosion/deposition rate in processes with mass entrainment at the basal surface. We are working on an explicit expression for $\mathcal{M}_{\text {int }}$ and will report the results in due time.

\section{SPECIAL CASE - ONE-DIMENSIONAL TWO-LAYER FLOW}

As an example we consider the flow without mass exchange at the layer interface over a one-dimensional slightly curved surface. That is, the basal surface is

$$
x_{1}=x, x_{2}=y, x_{3}=b(x), \Omega=O\left(\epsilon^{\gamma}\right) ;
$$

field quantities are independent of $y$, and $\boldsymbol{v}, \boldsymbol{u}, \boldsymbol{v}_{2}$ have negligibly small components in the direction $\mathrm{Ox}_{2}$ :

$$
\boldsymbol{v} \cdot \boldsymbol{i}_{2}=O\left(\epsilon^{1+\gamma}\right), \boldsymbol{u} \cdot \boldsymbol{i}_{2}=O\left(\epsilon^{1+\gamma}\right), \boldsymbol{v}_{2} \cdot \boldsymbol{i}_{2}=O\left(\epsilon^{1+\gamma}\right) .
$$

As parameters $\xi^{1}, \xi^{2}$ on the surface (108) we take

$$
\xi^{1}=s(x) \equiv \int_{x_{0}}^{x} \sqrt{1+(d b / d x)^{2}} d x^{\prime}, \xi^{2}=y,
$$

where $x_{0}=$ constant is such that the plane $x=x_{0}$ does not intersect the avalanche body at any moment. Clearly, $s(x)$ represents the arc length between $x_{0}$ and $x$ of the curve $x_{1}=x, x_{2}=$ constant, $x_{3}=b(x)$. Since corresponding to this parameterization we have $\boldsymbol{g}_{2}=$ $\tau_{2}+O\left(\epsilon^{1+\gamma}\right)=\boldsymbol{i}_{2}+O\left(\epsilon^{1+\gamma}\right)$, conditions (109) state that the mean velocities $\overline{\mathbf{v}}, \overline{\mathbf{u}}, \overline{\mathbf{v}}_{2}$ are of the form

$$
\begin{aligned}
& \overline{\mathbf{v}}=\left(V, O\left(\epsilon^{1+\gamma}\right)\right)^{T}, \overline{\mathbf{u}}=\left(U, O\left(\epsilon^{1+\gamma}\right)\right)^{T}, \\
& \overline{\mathbf{v}}_{2}=\left(V_{2}, O\left(\epsilon^{1+\gamma}\right)\right)^{T} .
\end{aligned}
$$

The shallow flow equations that we envisage are (90) (96) and $s_{\text {int }}=0$, and the closure relations are those from Sect. 4. Thus, we have (see [5])

$$
\begin{aligned}
& c=\sqrt{1-(d b / d s)^{2}}, \mathbf{s}=\left(\begin{array}{c}
d b / d s \\
0
\end{array}\right), \mathbf{F}=\left(\begin{array}{ll}
c & 0 \\
0 & 1
\end{array}\right), \\
& J_{0}=1, \mathbf{M}_{0}=\mathbf{I}, \Omega=\frac{1}{2 c} \frac{d^{2} b}{d s^{2}}, \\
& a_{s}=2 \Omega V^{2}, a_{f}=2 \Omega U^{2}, a_{2}=2 \Omega\left(V_{2}\right)^{2} .
\end{aligned}
$$

For the granular solid we assume model 2 or 3 (they coincide if the topography (108) is considered, see [5]), with the earth-pressure coefficient $\tilde{k}$ given by

$$
\begin{aligned}
& \tilde{k} \equiv \tilde{k}_{\text {act }}^{1} \quad \text { if } \quad \partial V / \partial s>0, \tilde{k} \equiv \tilde{k}_{\text {pass }}^{1} \text { if } \quad \partial V / \partial s<0, \\
& \tilde{k}_{\text {act/pass }}^{1} \equiv 2 \sec ^{2} \varphi-1 \mp 2 \sec \varphi \tan \varphi,
\end{aligned}
$$


where $\varphi=$ constant is the angle of internal friction. Then, noting that

$$
\begin{aligned}
& \left.\mathbf{p}_{s}\right|_{\xi=0}=\left((\tan \delta)\left(\left.p_{s}\right|_{\xi=0}\right)_{+} \operatorname{sgn} V, 0\right)^{T}, \\
& \left.\mathbf{p}_{f}\right|_{\xi=0}=\left(c_{\text {base }} \chi_{f}^{2}(1-\bar{v})|U| U, 0\right)^{T}, \\
& \left\{J\left[\left(-p_{2} \mathbf{M}+\mathbf{P}_{2}\right) \operatorname{Grad} h_{1}-\mathbf{p}_{2}\right]\right\}_{\xi=h_{1}} \\
& \quad=\left(-h \frac{\partial c h_{1}}{\partial s}-c_{\text {int }} \chi_{\text {int }}^{2}\left|V_{2}-V_{1}\right|\left(V_{2}-V_{1}\right), 0\right)^{T}, \\
& \frac{d c}{d s}=-2 \Omega \frac{d b}{d s}=O\left(\epsilon^{\gamma}\right),
\end{aligned}
$$

where $\left.p_{s}\right|_{\xi=0}$ is given by (82), $V_{1} \equiv \bar{v} V+(1-\bar{v}) U$, and $O\left(\epsilon^{2+\gamma}\right)$ terms have been neglected, after straightforward calculations we arrive at the following results.

- Equations for the solid constituent in the lower layer:

$$
\frac{\partial}{\partial t}\left\{h_{1} \bar{v}\right\}+\frac{\partial}{\partial s}\left\{h_{1} n_{1}^{(s)} \bar{v} V\right\}=0
$$

as the depth-integrated mass balance equation, and

$$
\begin{aligned}
& \frac{\partial}{\partial t}\left\{h_{1} n_{1}^{(s)} \bar{v} V\right\}+\frac{\partial}{\partial s}\left\{h _ { 1 } \overline { v } \left[n_{2}^{(s)} V^{2}+b+\right.\right. \\
& \left.\left.\tilde{k} c\left(\frac{1}{2} h_{1}+\frac{1}{c_{s f}} h\right)\right]\right\}=\bar{v}\left(b+\frac{1}{c_{s f}} c h\right) \frac{\partial h_{1}}{\partial s} \\
& +\frac{1}{c_{s f}} c_{i n t} \chi_{\text {int }}^{2} \overline{\mathrm{v}}\left|V_{2}-V_{1}\right|\left(V_{2}-V_{1}\right) \\
& -\tan \delta\left\{h_{1}\left(c+a_{s} m_{2}^{(s)}\right)+\frac{1}{c_{s f}} h\left(c+a_{2} m_{2}^{(2)}\right)\right\}_{+} \bar{v} \operatorname{sgn} V \\
& +h_{1}\left\{b+\frac{c h}{c_{s f}}+\frac{c h_{1}}{2\left(c_{s f} \overline{\mathrm{v}}+1-\overline{\mathrm{v}}\right)}\right\} \frac{\partial \overline{\mathrm{v}}}{\partial s}+c_{D} h_{1}(U-V),
\end{aligned}
$$

as the depth-integrated momentum balance equation.

- Equations for the fluid constituent in the lower layer:

$$
\frac{\partial}{\partial t}\left\{h_{1}(1-\bar{v})\right\}+\frac{\partial}{\partial s}\left\{h_{1}\left(1-n_{1}^{(f)} \bar{v}\right) U\right\}=0
$$

as the depth-integrated mass balance equation, and

$$
\begin{aligned}
& \frac{\partial}{\partial t}\left\{h_{1}\left(1-n_{1}^{(f)} \overline{\mathrm{v}}\right) U\right\} \\
& +\frac{\partial}{\partial s}\left\{h _ { 1 } \left[\left(m_{2}^{(f)}-n_{2}^{(f)} \overline{\mathrm{v}}\right) U^{2}+(1-\overline{\mathrm{v}}) b\right.\right. \\
& \left.\left.+(1-\overline{\mathrm{v}}) c\left(\frac{1}{2} h_{1}+h\right)\right]\right\}=(1-\overline{\mathrm{v}})(b+c h) \frac{\partial h_{1}}{\partial s} \\
& +c_{\text {int }} \chi_{\text {int }}^{2}(1-\overline{\mathrm{v}})\left|V_{2}-V_{1}\right|\left(V_{2}-V_{1}\right) \\
& -c_{\text {base }} \chi_{f}^{2}(1-\overline{\mathrm{v}})|U| U \\
& -h_{1}\left\{b+c h+\frac{c_{s f} c h_{1}}{2\left(c_{s f} \overline{\mathrm{v}}+1-\overline{\mathrm{v}}\right)}\right\} \frac{\partial \bar{v}}{\partial s}-c_{s f} c_{D} h_{1}(U-V),
\end{aligned}
$$

as the depth-integrated momentum balance equation.

- Equations for the upper layer:

$$
\frac{\partial h}{\partial t}+\frac{\partial}{\partial s}\left\{h V_{2}\right\}=0
$$

as the depth-integrated mass balance equation, and

$$
\begin{aligned}
& \frac{\partial}{\partial t}\left\{h V_{2}\right\}+\frac{\partial}{\partial s}\left\{h\left(m_{2}^{(2)} V_{2}^{2}+b+c h_{2}\right)\right\} \\
& =\left(b+c h_{2}\right) \frac{\partial h}{\partial s}-c_{i n t} \chi_{\text {int }}^{2}\left|V_{2}-V_{1}\right|\left(V_{2}-V_{1}\right),
\end{aligned}
$$

as the depth-integrated momentum balance equation.

Note that the curvature of the basal surface enters these equations only by means of the Coulomb basal friction stress, since the terms with $\partial c / \partial s=-2 \Omega \partial b / \partial s$ as a factor are negligibly small. However, the cosine of the angle between the vertical direction and the normal to the basal topography, $c$, may vary, in contrast to the case of an inclined plane, when it is constant.

\section{ON THE EQUILIBRIUM FREE SURFACE}

If a water layer is at rest, its free surface is horizontal, and hence a model describing the flow of such a layer should predict a horizontal free surface when the water stops to flow. In this paper we dealt with a two-layer system. Assuming that the upper layer consists of water, we are tempted to expect from the modeling equations to predict a horizontal free surface when the system is at rest. However, there are two basic underlying assumptions which we used throughout the paper: (i) at any moment the avalanching mass consists of a mixture layer overlain by a lighter fluid, also present as pore fluid in the mixture, and (ii) both layers are thin. Imagine, now, that this two-layer system is at rest. If the basal topography is only slightly curved, the previously mentioned assumptions (i) and (ii) very likely hold, and hence the modeling equations should indeed predict a horizontal free surface for the rest state. However, if the basal surface has large curvature, as in Fig. 4, the two-layer structure of the flowing mass is destroyed if there is not sufficient water in the upper layer; or, if there is sufficient water, the shallowness approximation is no longer fulfilled, as shown in Fig. 4.

Now we prove that the modeling equations describing the two thin immiscible/miscible layers, with water as the fluid present in both layers and Chézy-like friction at the layer interface, predict a horizontal free surface whenever the avalanching mass is at rest above a slightly curved basal surface, i.e., when

$$
\begin{aligned}
& \overline{\mathbf{v}}=\mathbf{0}, \quad \overline{\mathbf{u}}=\mathbf{0}, \quad \overline{\mathbf{v}}_{2}=\mathbf{0}, \\
& \frac{\partial \bar{v}}{\partial t}=0, \quad \frac{\partial h_{1}}{\partial t}=0, \quad \frac{\partial h_{2}}{\partial t}=0
\end{aligned}
$$

hold within a time interval, and $\mathcal{H}=O\left(\epsilon^{\gamma}\right)$. 


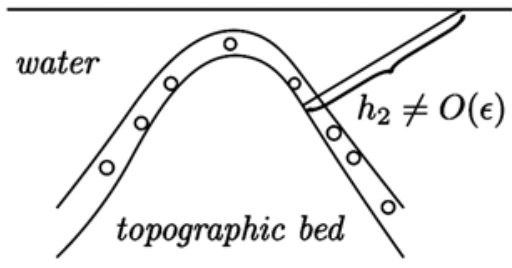

Fig. 4 Over-saturated mixture in equilibrium on basal topography with large curvature

We start from the depth-integrated momentum balance equation in the upper layer, (96). Using (110), (101) and (105) in (96) we deduce

$$
\mathbf{F M}_{0} \operatorname{Grad} c h_{2}+c \mathbf{s}=\mathbf{0} \text {. }
$$

Moreover, with $\mathbf{M}_{0}$ given by (5) and $\mathbf{F}^{-T} \mathrm{Grad} c h_{2}=$ $\operatorname{grad} c h_{2}$, this relation turns into

$$
\operatorname{grad} c h_{2}-\left(\operatorname{grad} c h_{2} \cdot \mathbf{s}\right) \mathbf{s}+c \mathbf{s}=\mathbf{0} .
$$

Taking the inner product of (111) with $\mathbf{s}$ and noting that $\mathbf{s} \cdot \mathbf{s}=1-c^{2}$, see (4), we find

$$
\operatorname{grad} c h_{2} \cdot \mathbf{s}=1-\frac{1}{c^{2}},
$$

which, when inserted into (111) together with relation $\mathbf{s}=c \operatorname{grad} b$, gives

$$
\operatorname{grad}\left(b+c h_{2}\right)=\mathbf{0},
$$

showing that the equilibrium free surface is horizontal. We mention that, keeping Grad (ch) in (96) instead of $c$ Grad $h$ (clearly, Grad $c=O\left(\epsilon^{\gamma}\right)$, see (89)), we could exactly show that the equilibrium free surface of the two-layer system, with water in the upper layer, is horizontal.

If the curvature of the basal surface is arbitrary, starting from (88) and using the same line of argument as for the case $\mathcal{H}=O\left(\epsilon^{\gamma}\right)$, one can prove that the height $b+c h_{2}$ of the equilibrium surface of the two-layer system, with water in the upper layer and Chézy friction at the layer interface, satisfies the condition

$$
\begin{aligned}
& \operatorname{grad}\left(b+c h_{2}\right)=\left(h_{1}+h_{2}\right) \\
& \quad \times\left\{\Omega \mathbf{I}-c^{3} \operatorname{grad}(\operatorname{grad} b)\right\} \operatorname{grad} b .
\end{aligned}
$$

The right-hand side of (112) can be zero only for particular elevation functions $b$. Therefore, for arbitrary topography the equilibrium free surface fails to predict horizontal equilibrium surfaces. The ordering approximations, as explained referring to Fig. 4, are the likely reason for this property.

\section{DISCUSSIONS AND CONCLUSIONS}

This paper is a continuation of the work by Luca et al. [27,5]. In [27] the three-dimensional modeling equations of a shallow saturated solid-fluid mixture flowing over arbitrary fixed topography have been derived, while in [5] the three-dimensional modeling equations for a shallow two-layer system, consisting of a one-constituent debris layer overlain by a lighter fluid layer, with the account of erosion/deposition processes, have been deduced. Here we let the lower debris layer be a solid-fluid mixture as in [27], and the upper layer a lighter fluid layer as in [5]. The following particulars are the outstanding features of the model:

- The sediment and the fluid in the lower layer, as well as the fluid in the upper layer, are treated as density-preserving continuous bodies, each moving with its own velocity. The mixture in the lower layer is saturated, with the solid volume fraction as a measure of the degree of saturation.

- At the basal surface, entrainment or deposition of material and lift-off are not considered.

- We neither assume any mass exchange at the free surface of the two-layer system, e.g. due to precipitation; so this surface is material. The free surface is also considered to be traction-free.

- The layer interface, defined as the upper surface of the granular material, deserved special attention.

- Irrespective of the fact whether there is fluid mass entrainment at the layer interface or not, the layer interface is material with respect to the solid.

- If the two layers are immiscible, the interface is also material with respect to the pore fluid and the fluid in the upper layer. Accordingly, three kinematic scalar restrictions express this fact.

- If the layers are miscible, the jump of fluid mass accounts for this mass flow. Thus, two kinematic scalar restrictions describe this case.

Important steps in the formulation of the topography adjusted governing equations are as follows:

- Dimensionless balances of mass of solid and fluid constituents, and kinematic and dynamic boundary conditions in curvilinear coordinates (Sect. 3.2).

- Reduction of these equations to a spatially twodimensional description by depth integration over the two layers with special consideration of the fluid mass exchange at the interface (Sect. 3.3).

- Performing a scaling analysis adjusted to considerations of shallowness of geometry and flow considerations (Sect. 3.4) and presentation of modeling equations applicable under small curvatures (Sect. 3.5).

- Closing the equations by a selection of constitutive relations for the stresses (Sect. 4.1) and discussing the interfacial fluid mass flux (Sect. 4.2).

The model equations were also given in spatially one-dimensional form - the dominant case treated in the literature (Sect. 5), and for the 2D case with small curvature it was demonstrated that the equilibrium configurations necessarily possess horizontal free surfaces.

\section{ACKNOWLEDGMENTS}

C.Y. Kuo and Y.C. Tai would like to acknowledge the financial support provided by National Science Council, 
Taiwan (Project No.: NSC 97-2221-E-260-018). The work of $\mathrm{KH}$ has been self-supported. We thank one referee for pointing out the work of Berzi et al. and a second referee for his/her very useful conducted review which improved the writing considerably.

\section{REFERENCES}

1. Larcan, E., Mambretti, S. and Pulecchi, M., "A Procedure for the Evaluation of Debris Flow Stratification. Monitoring, Simulation, Prevention and Remediation of Dense and Debris Flows," Transaction: Ecology and the Environement, 90, Eds. G. Lorenzini, C.A. Brebbis, D. Emmanouloudis, WIT Press (2006).

2. Takahashi, T., Debris Flow, Mechanics, Prediction and Countermeasures. Taylor \& Francis, London, (2007).

3. Fraccarollo, L. and Capart, H., "Riemann Wave Description of Erosional Dam-Break Flows," Journal of Fluid Mechanics, 461, pp. 183-228 (2002).

4. Morales de Luna, T., "A Saint Venant Model for Gravity Driven Shallow Water Flows with Variable Density and Compressibility Effects," Mathematical and Computer Modeling, 47, pp. 436-444 (2008).

5. Luca, I., Hutter, K., Kuo, C. Y. and Tai, Y. C., "Two-Layer Models for Shallow Avalanche Flows Over Arbitrary Variable Topography," International Journal of Advances in Engineering Sciences and Applied Mathematics, 1, pp. 99-121 (2009).

6. Fernández-Nieto, E. D., Bouchut, F., Bresch, D., Castro-Díaz, M. J. and Mangeney, A., "A New Savage-Hutter Type Model for Submarine Avalanches and Generated Tsunami," Journal of Computational Physics, 227, pp. 7720-7754 (2008).

7. Takahama, J., Fujita, Y., Hachiya, K. and Yoshino, K., "Application of Two Layer Simulation Model for Unifying Debris Flow and Sediment Sheet Flow and Its Improvement," Debris-Flow Hazards Mitigation: Mechanics, Prediction, and Assessment, Eds. Rickenmann \& Chen, Millpress, Rotterdam (2003).

8. Berzi, D. and Jenkins, J. T., "Approximate Analytical Solutions in a Model for Highly Concentrated Granular-Fluid Flows," Physics Review E, 78, 011304, (2008).

9. Berzi, D. and Jenkins, J. T., "Steady, Inclined Flows of Granular-Fluid Mixtures," Journal of Fluid Mechanics, 641, pp. 359-387 (2009).

10. Berzi, D., Jenkins, J. T. and Larcan, M., "Approximate Analytical Solutions in a Model for Highly Concentrated Granular-Fluid Flows," Advances in Geophysics, 52, pp. 103-138 (2010).

11. Hutter, K., Jöhnk, K. and Svendsen, B., “On Interfacial Transition Conditions in Two Phase Gravity Flow," ZAMP, 45, pp. 746-762 (1994).

12. Svendsen, B., Wu, T., Jöhnk, K. and Hutter, K., “On the Role of Mechanical Interactions in the SteadyState Gravity Flow of a Two-Constituent Mixture Down an Inclined Plane," Proceedings of the Royal
Society of London, A, 452, pp. 1189-1205 (1996).

13. Wu, T., Hutter, K. and Svendsen, B., "On Shear Flow of a Saturated Ice-Sediment Mixture with Thermodynamic Equilibrium Pressure and Momentum Exchange," Proceedings of the Royal Society of London, A, 454, pp. 71-88 (1998).

14. Sivakumaran, N. S., Tingsanchali, T. and Hosking, R. J., "Steady Shallow Flow Over Curved Beds," Journal of Fluid Mechanics, 128, pp. 469-487 (1983).

15. Dewals, B. J., Erpicum, S., Archambau, P., Detrembleur, S. and Pirotton, M., "Depth-Integrated Flow Modeling Taking Into Account Bottom Curvature," Journal of Hydraulic Research, 44, pp. 785-795 (2006).

16. Iverson, R. M., "The Physics of Debris Flows," Reviews of Geophysics, 35, pp. 245-296 (1997).

17. Pudasaini, S. P., Wang, Y. and Hutter, K., "Rapid Motions of Free Surface Avalanches Down Curved and Twisted Channels and Their Numerical Simulation," Philosophical Transactions of the Royal Society, A, 363, pp. 1551-1571 (2005).

18. Pudasaini, S. P., Wang, Y. and Hutter, K., "Modeling Debris Flows Down General Channels," Natural Hazards and Earth System Sciences, 5, pp. 799-819 (2005).

19. Bouchut, F. and Westdickenberg, M., "Gravity Driven Shallow Water Models for Arbitrary Topography," Communications in Mathematical Sciences, 2, pp. 359-389 (2004).

20. De Toni, S. and Scotton, P., "Two-Dimensional Mathematical and Numerical Model for the Dynamics of Granular Avalanches," Cold Regions Science and Technology, 43, pp. 36-48 (2005).

21. Bouchut, F., Fernández-Nieto, E. D., Mangeney, A. and Lagrée, P.-Y., "On New Erosion Models of Savage-Hutter Type for Avalanches," Acta Mechanica, 199, pp. 181-208 (2008).

22. Tai, Y. C. and Kuo, C. Y., "A New Model of Granular Flows Over General Topography with Erosion and Deposition," Acta Mechanica, 199, pp. 71-96 (2008).

23. Tai, Y. C. and Lin, Y. C., "A Focused View of the Behaviour of Granular Flows Down a Confined Inclined Chute Into the Horizontal Run-Out Zone," Physics of Fluids, 20, 123302 (2008).

24. Pelanti, M., Bouchut, F. and Mangeney, A., "A Roe-Type Scheme for Two-Phase Shallow Granular Flows Over Variable Topography," ESAIM: M2AN, 42, pp. 851-885 (2008).

25. Luca, I., Tai, Y. C. and Kuo, C. Y., "Non-Cartesian Topography Based Avalanche Equations and Approximations of Gravity Driven Flows of Ideal and Viscous Fluids," Mathematical Models and Methods in Applied Sciences, 19, pp. 127-171 (2009).

26. Luca, I., Hutter, K., Tai, Y. C. and Kuo, C. Y., "A Hierarchy of Avalanche Models on Arbitrary Topography," Acta Mechanica, 205, pp. 121-149

27. (2009)I., Tai, Y. C. and Kuo, C. Y., "Modeling Shallow Gravity-Driven Solid-Fluid Mixtures Over Arbi- 
trary Topography," Communications in Mathematical Sciences, 7, pp. 1-36 (2009).

28. Dressler, R. F., "New Nonlinear Shallow Flow Equations with Curvature," Journal of Hydraulic Research, 16, pp. 205-222 (1978).

29. Sivakumaran, N. S., Hosking, R. J. and Tingsanchali, T., "Steady Shallow Flow Over a Spillway," Journal of Fluid Mechanics, 111, pp. 411-420 (1981).

30. Egashira, S., Myamoto, K. and Itoh, T., "Constitutive Equations of Debris Flows and Their Applicability," Debris-Flow Hazards Mitigation: Mechanics, Prediction, and Assessment, Proceedings 1st International DFHM Conference, San Francisco, CA, USA, Ed. C.L. Chen, ASCE, pp. 340-349 (1997).

31. Iverson, R. M. and Denlinger, R. G., "Flow of Variably Fluidized Granular Masses Across ThreeDimensional Terrain, 1. Coulomb Mixture Theory," Journal of Geophysical Research, 106, pp. 537-552 (2001).

32. Egashira, S., Itoh, T. and Takeuchi, H., "Transition Mechanism of Debris Flows Over Rigid Bed to Over Erodible Bed," Physics and Chemistry of the Earth, B, 26, pp. 169-174 (2001).

33. Schneider, L. and Hutter, K., "Solid-Fluid Mixtures of Frictional Materials in Geophysical and Geotechnical Context," Advances in Geophysical and Environmental Mechanics and Mathematics, Springer Verlag, Berlin, Heidelberg, New York, 247p (2009).
34. Lien, H. P. and Tsai, F. W., "Sediment Concentration Distribution of Debris Flow," Journal of Hydraulic Engineering, DOI: 10.1061(ASCE)0733-9429(2003) 129:12(995) (2003).

35. Kuo, C. Y., Tai, Y. C., Bouchut, F., Mangeney, A., Pelanti, M., Chen, R. F. and Chang, K. J., "Simulation of Tsaoling Landslide, Taiwan, Based on Saint Venant Equations Over General Topography," Engineering Geology, 104, pp. 181-189 (2009).

36. Pitman, E. B. and Le, L., "A Two-Fluid Model for Avalanche and Debris Flows," Philosophical Transactions of the Royal Society, A, 363, pp. 1573-1601 (2005).

37. Pudasaini, S. P. and Hutter, K., Avalanche Dynamics, Berlin Heidelberg, New York (2007).

38. Truesdell, V. and Toupin, R., Handbuch der Physik, $I I I / 1$, Ed. S. Flügge, Springer Verlag, Berlin, Göttingen, Heidelberg (1960).

39. Truesdell, C., Rational Thermodynamics, McGrawHill Series in Modern Applied Mathematics, New York 1969, 2nd Ed., Springer, New York (1984).

(Manuscript received September 16, 2010, accepted for publication August 2, 2011.) 